\title{
Modulation of plasma and urine metabolome in colorectal cancer survivors consuming rice bran
}

\author{
Iman Zarei ${ }^{1}$, Renee C Oppel ${ }^{1}$, Erica C Borresen ${ }^{1}$, Regina J Brown ${ }^{2}$ and Elizabeth P Ryan ${ }^{1 *}$ \\ ${ }^{1}$ Department of Environmental and Radiological Health Sciences, College of Veterinary Medicine and Biomedical Sciences, Colorado State University, Fort Collins, \\ CO, 80523, USA \\ ${ }^{2}$ University of Colorado School of Medicine, Aurora, CO 80045, USA
}

\begin{abstract}
Rice bran has bioactive phytochemicals with cancer protective actions that involve metabolism by the host and the gut microbiome. Globally, colorectal cancer (CRC) is the third leading cause of cancer-related death and the increased incidence is largely attributed to poor dietary patterns, including low daily fiber intake. A dietary intervention trial was performed to investigate the impact of rice bran consumption on the plasma and urine metabolome of CRC survivors. Nineteen CRC survivors participated in a randomized-controlled trial that included consumption of heat-stabilized rice bran (30 g/day) or a control diet without rice bran for 4 weeks. A fasting plasma and first void of the morning urine sample were analyzed by non-targeted metabolomics using ultrahigh-performance liquid chromatographytandem mass spectrometry (UHPLC-MS/MS). After 4 weeks of either rice bran or control diets, 12 plasma and 16 urine metabolites were significantly different between the groups $(p \leq 0.05)$. Rice bran intake increased relative abundance of plasma mannose (1.373-fold) and beta-citrylglutamate (BCG) $(1.593-$ fold), as well as increased urine N-formylphenylalanine (2.191-fold) and dehydroisoandrosterone sulfate (DHEA-S) (4.488-fold). Diet affected metabolites, such as benzoate, mannose, eicosapentaenoate (20:5n3) (EPA), and N- formylphenylalanine have been previously reported for cancer protection and were identified from the rice bran food metabolome. Nutritional metabolome changes following increased consumption of whole grains such as rice bran warrants continued investigation for colon cancer control and prevention attributes as dietary biomarkers for positive effects are needed to reduce high risk for colorectal cancer recurrence.
\end{abstract}

\section{Introduction}

Despite major advances in cancer screening and treatment, colorectal cancer (CRC) remains the third leading cause of cancer-related death in the world [1] and in the United States [2]. Evidence supports that a plant-based, fiber-rich diet can increase longevity and lower the risk of chronic diseases including obesity $[3,4]$, cardiovascular disease [5,6], diabetes [7-9], and CRC [1013]. The American Institute for Cancer Research (AICR) estimated that almost half of U.S. CRC incidences can be prevented through appropriate lifestyle behaviors, including regular physical activity and a healthy diet comprised of fruits, vegetables, whole grains, and beans [14].

Rice bran is a functional food that has been recognized as globally accessible, but not well known as food that can prevent and control chronic diseases $[15,16]$. Bioactive food components from rice bran were identified for cancer protective functions related to energy metabolism [17-19], antioxidant [20], and direct anti-neoplastic activity $[21,22]$. Rice bran was examined as functional food in research for both CRC treatment and prevention [23-27]. Consumption of brown rice once per week was shown to reduce the incidence of adenomatous polyps by $40 \%$ [28] and numerous studies demonstrate the diverse nutritional and medicinal properties of rice bran bioactive compounds $[21,29,30]$. Given the chronic disease fighting properties established for rice bran from in vitro and animal studies, this food was studied in humans and showed feasibility to increase total daily fiber intake in adults $[15,26]$ and children [31]. Notably, rice bran was beneficial to modulate the human stool microbiome [26,27] and metabolome [25], which provided rationale to measure metabolic changes in the plasma and urine.

Metabolomics has been shown to be a promising tool for assessing dietary intakes as well as the influence of dietary patterns in relation to cancer risk [24,25]. Metabolomics is used to measure a suite of metabolites in foods [32] and biofluids [33]. This approach has provided valuable insight into the nutrition and health implications on the host metabolome [34]. Plasma and urine are relevant biological matrices for studying the effects of dietary intakes in humans that may suppress or alter colon carcinogenesis [35]. The objective of this study was to identify metabolites and metabolic pathways affected by rice bran consumption in a cohort of overweight and obese individuals at risk for colon cancer recurrence. We hypothesized that rice bran consumption favorably modulates multiple metabolic pathways in the plasma and urine associated with colorectal cancer prevention. Identification of bioactive compounds in the metabolite profiles may uncover candidate dietary biomarkers of intake and a health beneficial response to rice bran by the host.

*Correspondence to: Elizabeth P. Ryan, $\mathrm{PhD}$, Department of Environmental and Radiological Health Sciences, University of Colorado Cancer Center, Colorado State University/ Colorado School of Public Health, 200 W. Lake St., 1680 Campus Delivery, Fort Collins, CO 80523-1680, USA, Tel: 9704911536; Fax: 9704917569; E-mail: E.P.Ryan@colostate.edu

Key words: rice bran, metabolomics, colorectal cancer, plasma, urine, food metabolites

Received: March 13, 2019; Accepted: April 02, 2019; Published: April 05, 2019 


\section{Materials and methods}

\section{Study design and dietary intervention}

CRC survivors were recruited through the University of Colorado Health-North Cancer Center Network (Fort Collins, CO) for a 4-week, randomized-controlled, single-blinded dietary intervention trial as previously described $[15,25,26]$. Eligibility included: 1) no history of other malignancies, other than a CRC diagnosis, 2) more than four months post cancer treatment (e.g. chemotherapy or radiation), 3) no history of food allergies or major dietary restrictions, 4) not currently pregnant or lactating, 5) a non-smoker, 6) not taking antibiotics within the month prior to enrollment, and 7) no history of gallstones [15].

Participants who met eligibility criteria were randomized by body mass index (BMI), sex, and daily caloric intake. At the baseline, participants completed a 3-day food log recording all food and drink consumed on two weekdays and one weekend day and the food logs were analyzed using Nutritionist ProTM (Axxya Systems, Redmond, WA) as previously described [36]. Participants completed three study visits at baseline, week 2 , and week 4 when the fasting blood and first void urine samples were collected. Participants in the rice bran group consumed one snack and one meal daily that each contained $15 \mathrm{~g}$ of rice bran for a total consumption of $30 \mathrm{~g} /$ day. The control group was provided with macronutrient matched meals and snacks that did not include rice bran.

The Colorado State University Research Integrity and Compliance Review Board and the UCH-North Institutional Review Board approved this study protocol and informed consent (Protocol \#s 09$1530 \mathrm{H}$ and 10-1038, respectively). Figure 1 illustrates the 19 participants that completed the study called Bran Enriching Nutritional Eating for Intestinal health Trial (BENEFIT) (NCT01929122).

\section{Blood and urine sample collection for metabolomics}

The 19 participants that completed three study visits (baseline, 2 weeks, and week 4) provided fasting blood samples by venipuncture. Plasma was processed from whole blood collected in two $4 \mathrm{ml}$ ethylene-diamine-tetra-acetic acid (EDTA) blood collection tubes, and immediately placed on ice. Whole blood was centrifuged cold at 1500 rpm for 10 minutes, and $3 \mathrm{ml}$ of plasma was aliquoted from the sample and stored in $10 \mathrm{ml}$ tubes at $-80^{\circ} \mathrm{C}$ until processed for metabolomics by ultrahigh-performance liquid chromatography-tandem mass spectrometry (UPLC-MS/MS).

First void of the morning urine was self-collected by participants into study-coded specimen containers and transported to the lab within 2-hours of each study visit. Urine was aliquoted by lab personnel and stored at $-80^{\circ} \mathrm{C}$ until analyzed by UPLC-MS/MS. Osmolality normalization was applied to urine samples to correct for variance in fluid intake and urine solute concentrations. Osmolality of urine samples was measured using a Fiske ${ }^{\mathrm{TM}} 210$ Micro-Sample Osmometer. For normalization, the median-scaled ion peak data was divided by the osmolality value to obtain the osmo-corrected value.

\section{Plasma and urine sample extraction for metabolomics}

A non-targeted metabolite profiling for all samples was performed by Metabolon, Inc. (Durham, NC, USA). Global metabolic profiles were determined for each participant across both study groups. Each sample was entered into the Metabolon Laboratory Information Management System (LIMS) and was assigned a unique identifier that was associated with the original de-identified study code number. Extraction was performed on plasma and urine using $80 \%$ methanol under vigorous

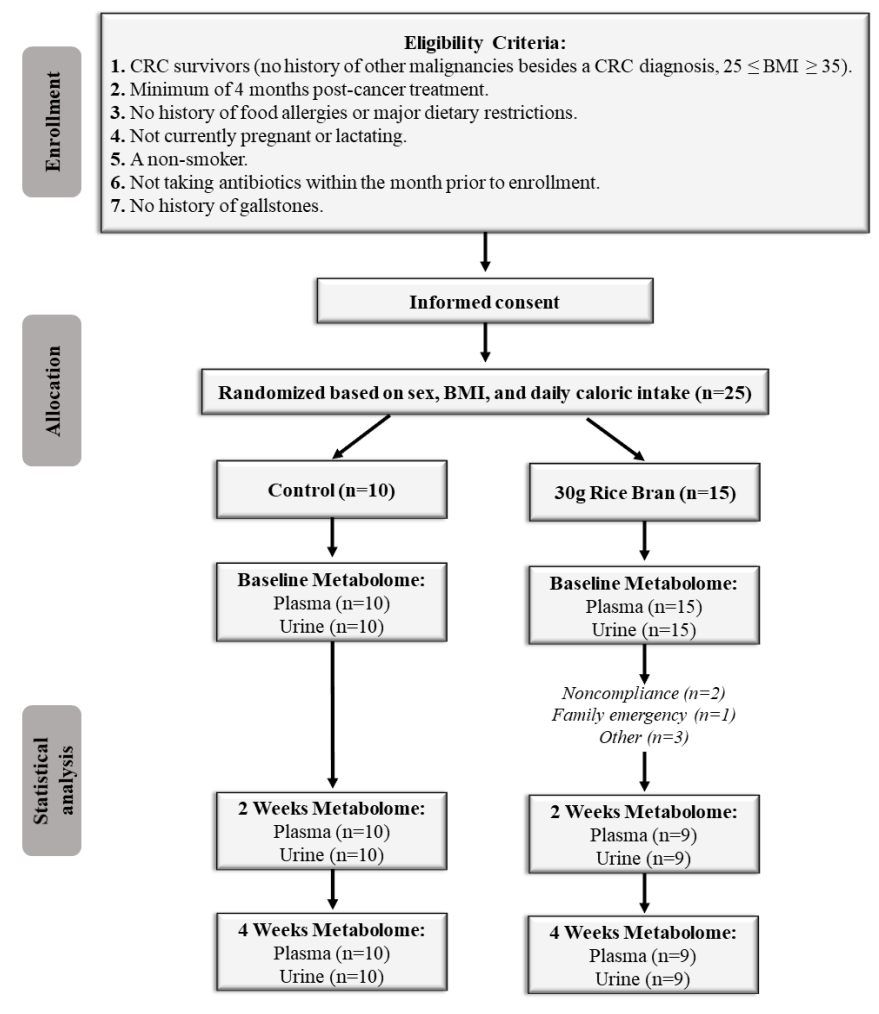

Figure 1. Study design for plasma and urine metabolome analysis in 19 colorectal cancer survivors (CRC) randomized to either control or rice bran intervention. The metabolomics data was analyzed in two comparisons; a) the control group and rice bran group had respective baselines, 2 weeks and 4 weeks post intervention analyses, b) rice bran group and control group were analyzed at 4 weeks post intervention

shaking for $2 \mathrm{~min}$ and was then centrifuged to dissociate metabolites bound to proteins. The resulting extracts from plasma and urine were divided into 3 fractions: 1 for analysis by UPLC-MS/MS with positive ion mode electrospray ionization (ESI), 1 for analysis by UPLC-MS/ MS with negative ion mode ESI, and 1 sample was reserved for backup. All samples (plasma and urine) were dried using a concentration evaporator (a TurboVap, Zymark), and then were stored overnight under liquid nitrogen before preparation for analysis [24].

\section{UPLC-MS/MS analysis}

Samples were analyzed using a Waters ACQUITY UPLC system coupled with a Thermo Scientific Q-Exactive high resolution/accurate mass spectrometer and interfaced with a heated electrospray ionization (HESI-II) source and Orbitrap mass analyzer operated at 35,000 mass resolution. Prior to analysis, acidic or basic UPLC-compatible solvents was used to re-suspend the dried samples. Eight quality control standards (known metabolites at fixed concentrations) were injected to each sample to guarantee injection and chromatographic consistency. The acidic re-suspension was measured using positive ion conditions, chromatographically optimized for either hydrophilic or hydrophobic compounds. for hydrophilic compounds, the extracts were gradient eluted from a C18 column (Waters UPLC BEH C18-2.1×100 mm, 1.7 $\mu \mathrm{m}$ ) using water and methanol, containing $0.05 \%$ perfluoropentanoic acid and $0.1 \%$ formic acid. For hydrophobic compounds, the extracts were gradient eluted from another but similar afore mentioned C18 column using methanol, acetonitrile, water, $0.05 \%$ perfluoropentanoic and $0.01 \%$ formic acid and was operated at an overall higher organic content. The basic solution was analyzed using either basic negative ion extracts or basic positive ion extracts. A C18 column (Waters UPLC 
BEH Amide $2.1 \times 150 \mathrm{~mm}, 1.7 \mu \mathrm{m}$ ) was utilized for both negative and positive ion extracts. The basic extracts were gradient eluted from the column using methanol, water, and $6.5 \mathrm{mM}$ ammonium bicarbonate at $\mathrm{pH}$ 8.0. The basic positive ion extracts were gradient eluted from the column with water, acetonitrile, and $10 \mathrm{mM}$ ammonium formate at $\mathrm{pH}$ 10.8. The mass spectrometry (MS) analysis utilized both MS and data-dependent MS/MS scans using dynamic exclusion. The scan range covered $70-1000 \mathrm{~m} / \mathrm{z}$ as previously described [24].

\section{Heat-stabilized rice bran metabolomics}

The food metabolome analysis used $100 \mathrm{mg}$ of heat-stabilized rice bran for non-targeted metabolite profiling. Rice bran was extracted with $80 \%$ methanol prior to UPLC-MS/MS and gas chromatographymass spectrometry (GC-MS) platforms [24,25]. The extracts that were allocated for GC-MS analysis, were fist vacuum-dried for 18 hours and then derivatized using bistrimethyl-silyltrifluoroacetamide. Chromatography separation on derivatized extracts was performed using 5\% diphenyl/95\% dimethyl polysiloxane fused silica column (20 $\mathrm{m} \times 0.18 \mathrm{~mm}$ ID; 0.18 um film thickness) with an applicable carrier gas as described previously [24,37]. To verify whether metabolites identified in plasma and/or urine originated from rice bran (exclusively or in part), all the plasma and urine metabolites identified in both dietary groups were cross-referenced with metabolites identified from the rice bran metabolome.

\section{Data extraction and compound identification}

Metabolon in-house peak detection and integration software was used to extract and process the raw data (quantitation is based on area under the curve from MS data). Standard industry approaches were used for MS peak detection, including minimum height, signalto-noise, width and area criteria. Compounds were compared to a library of purified standards and identified according to 3 criteria: the experimentally detected signature matching the accurate mass of the authentic standard within $8 \mathrm{ppm}$ (match to the National Institute of Standards and Technology library within \pm 0.005 atomic mass units), retention index match within a defined window (approximately 5 seconds), and the tandem mass spectrometry (MS/MS) forward and reverse scores [24].

\section{Metabolic pathway networks and analysis}

The raw abundance of all identified plasma and urine metabolites are shown in Supplementary Table 1. To visualize the networks of metabolic pathways from plasma and urine metabolites, pathway enrichment scores (PES) were generated using Cytoscape (Version 2.8.3). PES was calculated using the following equation:

\section{$\frac{k / m}{n / N}$}

In the equation, " $k$ " represent number of significant metabolites in a pathway $(p \leq 0 \cdot 05)$, " $m$ " the total number of identified metabolites in that pathway, "n" the total number of significant metabolites in the dataset, and " $N$ " the total number of identified metabolites in the complete dataset $[37,38]$. Metabolic pathways with PES less or greater than one indicated that the pathway contained 1 or more metabolites with a statistically-significant fold-difference (i.e., between rice bran group and control at 4 weeks) compared to all other pathways within the matrix.

\section{Statistical analyses}

Plasma and urine metabolite profiles were normalized by dividing the median of each metabolite raw abundance across the entire dataset to get the median-scaled relative abundance. A Welch's two-sample t-test and two-way analysis of variance (ANOVA) were used to compare the scaled values between groups (i.e., rice bran versus control). Repeated measures ANOVA was used to determine statistically-significant metabolites within groups over time. Metabolites that were statistically different at baseline between rice bran group and control were removed from the between-group analyses. False discovery rate ( $q$-value) was calculated to determine false discoveries common to multiplecomparison metabolomics studies. Fold-difference was determined by dividing the relative abundance of the metabolite in the rice bran group by the relative abundance of the metabolite in control. Fold-change was determined by dividing the relative abundance of the metabolite in the rice bran group at 2 or 4 weeks by the relative abundance at baseline (week 0). Significant metabolites had $p$-values of $\leq 0.05$ and $q$-values below the threshold of $\leq 0.10$.

\section{Results}

\section{Nutritional metabolome of control or rice bran groups}

The plasma metabolome had 854 metabolites and the urine metabolome had 703 metabolites with confirmed identification from 19 participants sampled at baseline, 2 weeks, and 4 weeks post intervention. The metabolites were classified as amino acids, carbohydrates, cofactors \& vitamins, energy metabolites, lipids, nucleotides, peptides, and xenobiotics. Supplementary Table 1 shows raw abundance of all metabolites organized into metabolic pathways for the rice bran and groups in both plasma and urine. All rice bran food metabolites that were also detected in the plasma and/or urine metabolome are listed in Supplementary Table 2. Food metabolomics of rice bran showed 453 unique metabolites and 103 rice bran metabolites were detected in plasma and/or urine after 2 or 4 weeks of consumption. Thus, up to $5.5 \%$ of the plasma metabolome and $9.7 \%$ of the urine metabolome could be classified as derived from rice bran phytochemicals.

\section{Dietary modulation of the plasma metabolome in CRC survivors}

Consumption of rice bran for 4 weeks resulted in significantmodulation of 11 metabolites including 8 lipids, 1 amino acid, 1 carbohydrate, and 1 xenobiotic compared to control $(n=19)$. These plasma metabolites with significant differences in the abundance between rice bran group and control are listed in Table 1 and visualized in Figure 2A $(p \leq 0.05)$. For all participants, data are presented as the mean fold-difference between rice bran group and control at 4 weeks. Among the 11 plasma metabolites with significant fold-differences in the rice bran group at 4 weeks compared to control, 2 metabolites (mannose and benzoate) were also identified in the rice bran food metabolome (footnote ${ }^{1}$ in Table 1). The 2 metabolites that increased in plasma of the rice bran group were $\beta$-citrylglutamate (BCG) (1.59-fold) and mannose (1.37-fold). The nine plasma metabolites with a significant decrease compared to control at 4 weeks were linoleoylcholine (0.24-fold), oleoylcholine (0.25-fold), palmitoylcholine (0.25-fold), dihomo-linolenoylcarnitine (C20:3) (DGLA) (0.62-fold), dihomolinolenoylcarnitine (C20:2) (0.67-fold), arachidonoylcholine (0.27fold), dihomo-linolenoyl-choline (0.25-fold), docosahexaenoylcholine ( 0.25 -fold), and benzoate ( 0.70 -fold) .

Table 2 lists metabolites significantly modulated in both dietary groups over time, with changes reported at 2- and 4-weeks post 
Table 1. Rice bran consumption showed different plasma metabolites at 4 weeks compared to control group

\begin{tabular}{|c|c|c|c|}
\hline \multirow[b]{2}{*}{ Metabolite } & \multirow[b]{2}{*}{ HMDB } & \multicolumn{2}{|c|}{ Fold-difference* } \\
\hline & & Rice Bran & $p$-value \\
\hline \multicolumn{4}{|l|}{ Amino Acid } \\
\hline beta-citrylglutamate (BCG) & HMDB13220 & 1.60 & 0.039 \\
\hline Betaine $^{1}$ & HMDB00043 & 0.80 & 0.057 \\
\hline \multicolumn{4}{|l|}{ Carbohydrate } \\
\hline Mannose $^{1}$ & HMDB00169 & 1.37 & 0.022 \\
\hline \multicolumn{4}{|l|}{ Lipids } \\
\hline Arachidonoylcholine & - & 0.27 & 0.026 \\
\hline Dihomo-linolenoylcarnitine (20:3n3 or 6 ) (DGLA) & - & 0.62 & 0.033 \\
\hline Dihomo-linolenoyl-choline & - & 0.25 & 0.029 \\
\hline Dihomo-linoleoylcarnitine (C20:2) & - & 0.67 & 0.026 \\
\hline Docosahexaenoylcholine & - & 0.25 & 0.010 \\
\hline Linoleoylcarnitine (C18:2) & HMDB06469 & 0.71 & 0.064 \\
\hline Linoleoylcholine & - & 0.24 & 0.028 \\
\hline Oleoylcholine & - & 0.25 & 0.016 \\
\hline Palmitoylcholine & - & 0.25 & 0.024 \\
\hline \multicolumn{4}{|l|}{ Xenobiotics } \\
\hline Benzoate $^{1}$ & HMDB01870 & 0.69 & 0.046 \\
\hline
\end{tabular}

GPC: Glycerophosphocholine; HMDB: Human metabolome database

${ }^{1}$ Metabolite also identified from the rice bran metabolome

*Values presented are fold-difference of the mean relative abundance between rice bran group compared to control at 4 weeks ( $p \leq 0.05$ ). Statistically-significantly increased fold-differences are highlighted in red and statistically-significantly decreased fold-differences are highlighted in blue. Light blue and light red coloring represent metabolites and pathways that showed trends towards significance $(0.05 \leq \mathrm{p} \leq 0.10)$

Table 2. Modulated plasma metabolites following control or rice bran consumption at 2 and 4 weeks post intervention when compared to baseline

\begin{tabular}{|c|c|c|c|c|c|c|c|c|c|c|}
\hline & \multirow{3}{*}{ Metabolite } & \multirow{3}{*}{ HMDB } & \multicolumn{8}{|c|}{ Fold-change* } \\
\hline & & & \multicolumn{4}{|c|}{ Control group } & \multicolumn{4}{|c|}{ Rice bran group } \\
\hline & & & 2wk/ 0wk & p-value & $4 w k / 0 w k$ & p-value & 2wk/ 0wk & p-value & $4 w k / 0 w k$ & p-value \\
\hline \multicolumn{11}{|l|}{ Amino acid } \\
\hline Alanine and Aspartate & Alanine $^{1}$ & HMDB00161 & 0.94 & 0.119 & 0.99 & 0.731 & 0.96 & 0.324 & 0.92 & 0.049 \\
\hline Creatine & Creatine $^{1}$ & HMDB00064 & 0.99 & 0.802 & 0.97 & 0.465 & 0.87 & 0.046 & 0.97 & 0.526 \\
\hline Glutamate & $\beta$-citrylglutamate (BCG) & - & 1.01 & 0.584 & 0.82 & 0.054 & 1.22 & 0.862 & 1.67 & 0.037 \\
\hline Glutathione & Cysteinylglycine & HMDB00078 & 0.91 & 0.040 & 0.93 & 0.449 & 1.21 & 0.496 & 1.12 & 0.797 \\
\hline \multirow{3}{*}{ Leucine, Isoleucine and Valine } & 3-methyl-2-oxovalerate ${ }^{1}$ & HMDB03736 & 0.90 & 0.028 & 0.96 & 0.368 & 0.98 & 0.695 & 1.01 & 0.992 \\
\hline & Isoleucine $^{1}$ & HMDB00172 & 0.95 & 0.050 & 1.00 & 0.870 & 0.99 & 0.831 & 1.03 & 0.572 \\
\hline & Methylsuccinoylcarnitine & - & 1.53 & 0.028 & 1.30 & 0.346 & 1.00 & 0.807 & 1.01 & 0.874 \\
\hline \multirow{2}{*}{ Lysine } & Glutarate (pentanedioate) $^{1}$ & HMDB00661 & 1.30 & 0.431 & 1.04 & 0.909 & 0.77 & 0.046 & 1.07 & 0.596 \\
\hline & $\mathrm{N}$-acetyl-cadaverine & & 1.84 & 0.116 & 1.64 & 0.114 & 0.57 & 0.012 & 0.68 & 0.052 \\
\hline \multirow{2}{*}{$\begin{array}{l}\text { Methionine, Cysteine, SAM and } \\
\text { Taurine }\end{array}$} & Cysteine $^{1}$ & HMDB00574 & 0.86 & 0.034 & 0.94 & 0.494 & 1.13 & 0.316 & 1.25 & 0.075 \\
\hline & S-methylcysteine sulfoxide & - & 1.53 & 0.033 & 1.66 & 0.008 & 1.32 & 0.164 & 1.32 & 0.239 \\
\hline \multirow{3}{*}{ Phenylalanine and Tyrosine } & 4-hydroxyphenylacetate & HMDB00020 & 2.88 & 0.268 & 3.17 & 0.116 & 0.59 & 0.025 & 1.47 & 0.833 \\
\hline & Gentisate $^{1}$ & HMDB00152 & 5.89 & 0.007 & 4.68 & 0.031 & 1.71 & 0.239 & 1.47 & 0.747 \\
\hline & Phenol sulfate & HMDB60015 & 1.26 & 0.307 & 1.72 & 0.060 & 1.56 & 0.078 & 1.59 & 0.048 \\
\hline Polyamine & $\mathrm{N}$-acetylputrescine ${ }^{1}$ & HMDB02064 & 0.95 & 0.321 & 1.01 & 0.981 & 0.91 & 0.111 & 0.89 & 0.046 \\
\hline \multirow{2}{*}{ Tryptophan } & 3-indoxyl sulfate & HMDB00682 & 1.03 & 0.850 & 1.13 & 0.697 & 0.75 & 0.012 & 0.87 & 0.117 \\
\hline & Indoleacetylglutamine & HMDB13240 & 1.71 & 0.144 & 1.28 & 0.810 & 0.74 & 0.044 & 1.14 & 0.736 \\
\hline \multirow{2}{*}{ Urea cycle; Arginine and Proline } & 2-oxoarginine & HMDB04225 & 1.37 & 0.656 & 1.34 & 0.409 & 0.70 & 0.040 & 0.91 & 0.224 \\
\hline & Pro-hydroxy-pro & HMDB06695 & 1.00 & 0.639 & 0.83 & 0.037 & 1.10 & 0.561 & 1.09 & 0.809 \\
\hline \multicolumn{11}{|l|}{ Carbohydrate } \\
\hline $\begin{array}{l}\text { Fructose, Mannose and } \\
\text { Galactose }\end{array}$ & Mannose $^{1}$ & HMDB00169 & 0.87 & 0.049 & 0.92 & 0.222 & 1.08 & 0.396 & 1.08 & 0.397 \\
\hline \multirow{2}{*}{ Pentose } & Arabinose $^{1}$ & HMDB00646 & 1.03 & 0.213 & 1.39 & 0.463 & 0.59 & 0.036 & 1.00 & 0.197 \\
\hline & Sedoheptulose & HMDB03219 & 1.23 & 0.849 & 1.17 & 0.943 & 2.93 & 0.017 & 1.41 & 0.498 \\
\hline \multicolumn{11}{|l|}{ Cofactors \& vitamins } \\
\hline \multirow{3}{*}{ Hemoglobin and Porphyrin } & Bilirubin $[\mathrm{E}, \mathrm{E}]$ & - & 0.72 & 0.002 & 0.73 & 0.018 & 0.99 & 0.546 & 0.93 & 0.335 \\
\hline & Bilirubin $[\mathrm{Z}, \mathrm{Z}]$ & HMDB00054 & 0.70 & 0.034 & 0.98 & 0.270 & 0.92 & 0.482 & 0.56 & 0.098 \\
\hline & Heme & HMDB03178 & 2.49 & 0.711 & 1.13 & 0.724 & 2.32 & 0.173 & 4.06 & 0.009 \\
\hline \multirow{4}{*}{ Tocopherol } & $\alpha$-CEHC sulfate & - & 0.71 & 0.040 & 1.05 & 0.963 & 0.72 & 0.111 & 0.76 & 0.108 \\
\hline & $\delta$-tocopherol ${ }^{1}$ & HMDB02902 & 0.67 & 0.031 & 0.92 & 0.205 & 0.97 & 0.218 & 0.89 & 0.406 \\
\hline & $\gamma$-CEHC & HMDB01931 & 0.81 & 0.048 & 1.12 & 0.378 & 0.62 & 0.004 & 0.71 & 0.019 \\
\hline & $\gamma$-CEHC glucuronide & - & 0.78 & 0.022 & 1.59 & 0.874 & 0.55 & 0.014 & 0.66 & 0.023 \\
\hline
\end{tabular}




\begin{tabular}{|c|c|c|c|c|c|c|c|c|c|c|}
\hline & \multirow{3}{*}{ Metabolite } & \multirow{3}{*}{ HMDB } & \multicolumn{8}{|c|}{ Fold-change* } \\
\hline & & & \multicolumn{4}{|c|}{ Control group } & \multicolumn{4}{|c|}{ Rice bran group } \\
\hline & & & $2 w k / 0 w k$ & p-value & $4 \mathrm{wk} / 0 \mathrm{wk}$ & p-value & $2 w k / 0 w k$ & p-value & 4 wk/ 0wk & p-value \\
\hline \multicolumn{11}{|l|}{ Energy } \\
\hline TCA Cycle & Succinylcarnitine (C4-DC) & - & 1.51 & 0.039 & 1.05 & 0.646 & 1.02 & 0.884 & 0.96 & 0.553 \\
\hline \multicolumn{11}{|l|}{ Lipid } \\
\hline \multirow{2}{*}{ Ceramides } & $\begin{array}{l}\text { Ceramide (d18:2/24:1, } \\
\text { d18:1/24:2) }\end{array}$ & - & 1.31 & 0.006 & 1.18 & 0.072 & 1.03 & 0.809 & 0.94 & 0.207 \\
\hline & $\begin{array}{l}\text { Glycosyl ceramide (d18:2/24:1, } \\
\text { d18:1/24:2) }\end{array}$ & - & 1.03 & 0.993 & 1.11 & 0.293 & 1.07 & 0.490 & 0.86 & 0.030 \\
\hline \multirow{2}{*}{ Diacylglycerol } & $\begin{array}{l}\text { Palmitoleoyl-oleoyl-glycerol } \\
(16: 1 / 18: 1)[2]\end{array}$ & - & 0.92 & 0.552 & 0.97 & 0.478 & 0.72 & 0.026 & 1.08 & 0.613 \\
\hline & $\begin{array}{l}\text { Palmitoyl-linoleoyl-glycerol } \\
(16: 0 / 18: 2)[1]\end{array}$ & $\begin{array}{l}\text { HMDB05207/ } \\
\text { HMDB07103 }\end{array}$ & 0.83 & 0.170 & 1.07 & 0.768 & 0.71 & 0.023 & 1.25 & 0.888 \\
\hline Endocannabinoid & N-oleoyltaurine & - & 1.36 & 0.716 & 1.25 & 0.273 & 1.00 & 0.432 & 0.65 & 0.046 \\
\hline \multirow{4}{*}{ Fatty Acid (Acyl Choline) } & Linoleoylcholine & - & 2.06 & 0.682 & 2.31 & 0.159 & 0.92 & 0.187 & 0.37 & 0.008 \\
\hline & Oleoylcholine & - & 2.05 & 0.605 & 2.17 & 0.184 & 0.92 & 0.207 & 0.38 & 0.006 \\
\hline & Palmitoylcholine & - & 1.95 & 0.666 & 2.24 & 0.197 & 0.95 & 0.223 & 0.39 & 0.009 \\
\hline & Stearoylcholine & - & 2.36 & 0.605 & 2.63 & 0.380 & 0.89 & 0.110 & 0.34 & 0.005 \\
\hline \multirow{10}{*}{ Fatty Acid (Acyl Carnitine) } & 3-hydroxybutyrylcarnitine & - & 1.01 & 0.262 & 0.84 & 0.046 & 1.26 & 0.216 & 1.19 & 0.525 \\
\hline & Arachidonoylcarnitine (C20:4) & - & 1.11 & 0.296 & 1.10 & 0.329 & 0.98 & 0.753 & 0.84 & 0.031 \\
\hline & $\begin{array}{l}\text { Dihomo-linolenoylcarnitine } \\
\text { (20:3n3 or 6) (DGLA) }\end{array}$ & - & 1.10 & 0.341 & 1.12 & 0.213 & 1.02 & 0.906 & 0.87 & 0.040 \\
\hline & Eicosenoylcarnitine (C20:1) & - & 1.11 & 0.272 & 1.07 & 0.467 & 1.22 & 0.046 & 1.00 & 0.841 \\
\hline & Erucoylcarnitine (C22:1) & - & 1.01 & 0.702 & 1.10 & 0.329 & 1.33 & 0.008 & 1.04 & 0.896 \\
\hline & Linolenoylcarnitine (C18:3) & - & 1.10 & 0.592 & 1.06 & 0.622 & 1.02 & 0.866 & 0.82 & 0.029 \\
\hline & Linoleoylcarnitine (C18:2) & HMDB06469 & 1.10 & 0.371 & 1.08 & 0.417 & 1.02 & 0.894 & 0.85 & 0.024 \\
\hline & Oleoylcarnitine (C18:1) & HMDB05065 & 1.12 & 0.262 & 1.05 & 0.574 & 1.23 & 0.018 & 1.00 & 0.800 \\
\hline & $\begin{array}{l}\text { Pimeloylcarnitine/3- } \\
\text { methyladipoylcarnitine (C7-DC) }\end{array}$ & - & 1.10 & 0.493 & 1.28 & 0.215 & 1.90 & 0.003 & 1.42 & 0.101 \\
\hline & Suberoylcarnitine (C8-DC) & - & 1.04 & 0.736 & 0.95 & 0.627 & 2.03 & 0.021 & 1.52 & 0.128 \\
\hline Fatty Acid, Amino & 2-aminoheptanoate ${ }^{1}$ & - & 1.02 & 0.741 & 1.18 & 0.457 & 1.50 & 0.004 & 1.22 & 0.162 \\
\hline Fatty Acid, Dicarboxylate & 3-methyladipate ${ }^{1}$ & HMDB00555 & 1.77 & 0.451 & 1.58 & 0.878 & 1.17 & 0.784 & 1.11 & 0.046 \\
\hline \multirow{2}{*}{ Fatty Acid, Monohydroxy } & 13-HODE + 9-HODE ${ }^{1}$ & - & 2.02 & 0.062 & 2.07 & 0.030 & 1.69 & 0.734 & 1.13 & 0.916 \\
\hline & 16-hydroxypalmitate & HMDB06294 & 0.94 & 0.313 & 1.08 & 0.520 & 1.31 & 0.038 & 0.98 & 0.702 \\
\hline \multirow{12}{*}{ Lysolipid } & 1-arachidonoyl-GPC (20:4n6) & HMDB10395 & 1.11 & 0.396 & 1.24 & 0.037 & 0.97 & 0.634 & 0.88 & 0.139 \\
\hline & 1-linolenoyl-GPC (18:3) & - & 1.30 & 0.080 & 1.35 & 0.023 & 0.76 & 0.005 & 0.75 & 0.006 \\
\hline & 1-linoleoyl-GPC (18:2) & HMDB10386 & 1.16 & 0.242 & 1.27 & 0.017 & 0.93 & 0.204 & 0.88 & 0.103 \\
\hline & 1-linoleoyl-GPE (18:2) & HMDB11507 & 1.23 & 0.096 & 1.29 & 0.034 & 0.95 & 0.269 & 0.90 & 0.168 \\
\hline & 1-oleoyl-GPC (18:1) & HMDB02815 & 1.12 & 0.120 & 1.19 & 0.014 & 0.98 & 0.604 & 0.92 & 0.155 \\
\hline & 1-palmitoleoyl-GPC (16:1) & HMDB10383 & 1.20 & 0.018 & 1.29 & 0.001 & 0.95 & 0.396 & 0.90 & 0.086 \\
\hline & 1-palmitoyl-GPC (16:0) & HMDB10382 & 1.07 & 0.285 & 1.11 & 0.032 & 0.91 & 0.056 & 0.93 & 0.079 \\
\hline & 1-palmitoyl-GPG (16:0) & & 1.23 & 0.761 & 1.32 & 0.884 & 0.80 & 0.212 & 0.65 & 0.024 \\
\hline & 1-stearoyl-GPC (18:0) & HMDB10384 & 1.14 & 0.152 & 1.20 & 0.020 & 0.87 & 0.040 & 0.93 & 0.158 \\
\hline & 1-stearoyl-GPI (18:0) & HMDB61696 & 1.13 & 0.520 & 1.41 & 0.036 & 1.08 & 0.908 & 0.86 & 0.209 \\
\hline & 2-palmitoleoyl-GPC (16:1) & - & 1.61 & 0.239 & 2.72 & 0.008 & 0.75 & 0.048 & 0.90 & 0.471 \\
\hline & 2-palmitoyl-GPC (16:0) & HMDB61702 & 0.98 & 0.535 & 1.17 & 0.155 & 0.84 & 0.047 & 0.90 & 0.169 \\
\hline Mevalonate & 3-hydroxy-3-methylglutarate ${ }^{1}$ & HMDB00355 & 0.75 & 0.030 & 0.85 & 0.168 & 1.24 & 0.333 & 1.09 & 0.961 \\
\hline Monoacylglycerol & 2-myristoylglycerol (14:0) & - & 2.73 & 0.041 & 1.52 & 0.329 & 2.17 & 0.674 & 0.80 & 0.152 \\
\hline \multirow{8}{*}{ Phospholipid } & $\begin{array}{l}\text { 1-linoleoyl-2-arachidonoyl-GPC } \\
(18: 2 / 20: 4 \mathrm{n} 6)\end{array}$ & - & 1.13 & 0.162 & 1.11 & 0.151 & 0.88 & 0.057 & 0.88 & 0.046 \\
\hline & $\begin{array}{l}\text { 1-linoleoyl-2-linolenoyl-GPC } \\
(18: 2 / 18: 3)\end{array}$ & - & 1.24 & 0.550 & 1.23 & 0.475 & 0.81 & 0.068 & 0.70 & 0.009 \\
\hline & $\begin{array}{l}\text { 1-palmitoleoyl-2-linolenoyl-GPC } \\
\text { (16:1/18:3) }\end{array}$ & - & 1.14 & 0.375 & 1.30 & 0.058 & 0.92 & 0.354 & 0.77 & 0.012 \\
\hline & $\begin{array}{l}\text { 1-palmitoyl-2-arachidonoyl-GPI } \\
\text { (16:0/20:4) }\end{array}$ & - & 1.23 & 0.027 & 1.19 & 0.062 & 0.94 & 0.392 & 0.92 & 0.225 \\
\hline & $\begin{array}{l}\text { 1-stearoyl-2-linoleoyl-GPI } \\
(18: 0 / 18: 2)\end{array}$ & - & 1.09 & 0.381 & 1.09 & 0.319 & 0.87 & 0.066 & 0.85 & 0.032 \\
\hline & Arachidonoylcholine & - & 1.65 & 0.927 & 2.06 & 0.227 & 0.95 & 0.244 & 0.36 & 0.007 \\
\hline & Dihomo-linolenoyl-choline & - & 2.30 & 0.416 & 2.75 & 0.125 & 0.88 & 0.119 & 0.33 & 0.003 \\
\hline & Docosahexaenoylcholine & - & 2.02 & 0.352 & 2.29 & 0.110 & 1.05 & 0.283 & 0.36 & 0.001 \\
\hline
\end{tabular}




\begin{tabular}{|c|c|c|c|c|c|c|c|c|c|c|}
\hline & \multirow{3}{*}{ Metabolite } & \multirow{3}{*}{ HMDB } & \multicolumn{8}{|c|}{ Fold-change* } \\
\hline & & & \multicolumn{4}{|c|}{ Control group } & \multicolumn{4}{|c|}{ Rice bran group } \\
\hline & & & 2wk/ 0wk & p-value & $4 w k / 0 w k$ & p-value & $2 w k / 0 w k$ & p-value & 4wk/ 0wk & p-value \\
\hline \multirow{4}{*}{ Plasmalogen } & $\begin{array}{l}\text { 1-(1-enyl-palmitoyl)-2-linoleoyl- } \\
\text { GPE (P-16:0/18:2) }\end{array}$ & - & 0.91 & 0.215 & 1.02 & 0.837 & 0.83 & 0.037 & 0.94 & 0.375 \\
\hline & $\begin{array}{l}\text { 1-(1-enyl-palmitoyl)-2-oleoyl- } \\
\text { GPC (P-16:0/18:1) }\end{array}$ & - & 1.00 & 0.789 & 0.97 & 0.442 & 1.14 & 0.050 & 0.95 & 0.343 \\
\hline & $\begin{array}{l}\text { 1-(1-enyl-palmitoyl)-2- } \\
\text { palmitoleoyl-GPC (P-16:0/16:1) }\end{array}$ & - & 0.92 & 0.090 & 0.92 & 0.073 & 1.15 & 0.047 & 0.93 & 0.173 \\
\hline & $\begin{array}{l}\text { 1-(1-enyl-palmitoyl)-2-palmitoyl- } \\
\text { GPC (P-16:0/16:0) }\end{array}$ & - & 0.97 & 0.525 & 0.96 & 0.464 & 1.16 & 0.042 & 0.95 & 0.351 \\
\hline $\begin{array}{l}\text { Polyunsaturated Fatty Acid (n3 } \\
\text { and n6) }\end{array}$ & Docosatrienoate (22:3n3) & HMDB02823 & 1.40 & 0.676 & 1.42 & 0.844 & 0.68 & 0.027 & 1.57 & 0.792 \\
\hline \multirow{2}{*}{ Primary Bile Acid } & Cholate & HMDB00619 & 0.93 & 0.109 & 1.23 & 0.599 & 0.71 & 0.008 & 0.86 & 0.336 \\
\hline & Glycocholate glucuronide & - & 1.41 & 0.992 & 1.07 & 0.932 & 1.02 & 0.508 & 0.82 & 0.006 \\
\hline \multirow{5}{*}{ Secondary Bile Acid } & Deoxycholate $^{1}$ & HMDB00626 & 1.07 & 0.479 & 1.35 & 0.290 & 0.61 & 0.002 & 0.82 & 0.155 \\
\hline & Glycocholenate sulfate & - & 0.81 & 0.023 & 0.90 & 0.240 & 0.93 & 0.403 & 0.88 & 0.144 \\
\hline & Isoursodeoxycholate & HMDB00686 & 1.09 & 0.444 & 1.16 & 0.820 & 0.75 & 0.031 & 1.01 & 0.625 \\
\hline & Taurocholenate sulfate & - & 0.85 & 0.089 & 0.86 & 0.164 & 1.50 & 0.040 & 1.05 & 0.984 \\
\hline & Ursodeoxycholate & HMDB00946 & 1.39 & 0.188 & 1.79 & 0.822 & 0.61 & 0.005 & 0.71 & 0.092 \\
\hline \multirow{8}{*}{ Sphingolipid } & $\begin{array}{l}\text { lactosyl-N-palmitoyl-sphingosine } \\
\text { (d18:1/16:0) }\end{array}$ & - & 1.06 & 0.551 & 1.05 & 0.647 & 1.20 & 0.023 & 0.94 & 0.271 \\
\hline & $\begin{array}{l}\text { N-nervonoyl- } \\
\text { hexadecasphingosine } \\
\text { (d16:1/24:1) }\end{array}$ & - & 1.37 & 0.010 & 1.32 & 0.025 & 1.07 & 0.586 & 1.01 & 0.494 \\
\hline & $\begin{array}{l}\text { Sphingomyelin (d18:1/14:0, } \\
\text { d16:1/16:0) }\end{array}$ & - & 1.04 & 0.429 & 1.09 & 0.037 & 1.01 & 0.835 & 0.99 & 0.653 \\
\hline & $\begin{array}{l}\text { Sphingomyelin (d18:1/17:0, } \\
\text { d17:1/18:0, d19:1/16:0) }\end{array}$ & - & 1.05 & 0.583 & 1.09 & 0.276 & 1.19 & 0.009 & 0.98 & 0.596 \\
\hline & $\begin{array}{l}\text { Sphingomyelin (d18:1/18:1, } \\
\text { d18:2/18:0) }\end{array}$ & - & 1.05 & 0.548 & 1.10 & 0.191 & 1.14 & 0.045 & 1.00 & 0.885 \\
\hline & $\begin{array}{l}\text { Sphingomyelin (d18:1/22:2, } \\
\text { d18:2/22:1, d16:1/24:2) }\end{array}$ & - & 1.10 & 0.554 & 1.12 & 0.341 & 1.24 & 0.045 & 0.92 & 0.213 \\
\hline & Sphingomyelin (d18:2/23:1) & - & 1.12 & 0.275 & 1.16 & 0.122 & 1.26 & 0.011 & 0.95 & 0.333 \\
\hline & Sphingomyelin (d18:2/24:2) & - & 1.13 & 0.416 & 1.11 & 0.406 & 1.26 & 0.039 & 0.93 & 0.256 \\
\hline \multirow{3}{*}{ Steroid } & 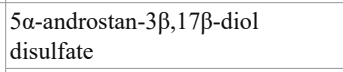 & HMDB00493 & 0.71 & 0.034 & 0.79 & 0.155 & 0.97 & 0.818 & 0.92 & 0.567 \\
\hline & Cortisone & HMDB02802 & 0.83 & 0.022 & 0.92 & 0.267 & 0.97 & 0.712 & 0.96 & 0.627 \\
\hline & Epiandrosterone sulfate & HMDB00365 & 0.60 & 0.043 & 0.60 & 0.044 & 0.64 & 0.099 & 0.71 & 0.178 \\
\hline \multirow{2}{*}{ Sterol } & $3 \beta, 7 \alpha$-dihydroxy-5-cholestenoate & - & 0.92 & 0.160 & 0.89 & 0.036 & 0.87 & 0.014 & 0.84 & 0.005 \\
\hline & 4-cholesten-3-one ${ }^{1}$ & HMDB00921 & 0.94 & 0.190 & 0.95 & 0.353 & 1.05 & 0.825 & 1.20 & 0.031 \\
\hline \multicolumn{11}{|l|}{ Nucleotide } \\
\hline \multirow{2}{*}{ Purine, Guanine containing } & 7-methylguanine ${ }^{1}$ & HMDB00897 & 0.92 & 0.032 & 0.92 & 0.032 & 1.02 & 0.685 & 0.98 & 0.584 \\
\hline & N2, N2-dimethylguanosine ${ }^{1}$ & HMDB04824 & 0.96 & 0.350 & 0.97 & 0.507 & 0.92 & 0.061 & 0.89 & 0.008 \\
\hline Pyrimidine, Cytidine containing & 3-methylcytidine & - & 0.83 & 0.003 & 0.86 & 0.028 & 0.91 & 0.137 & 1.06 & 0.507 \\
\hline \multicolumn{11}{|c|}{ Peptide } \\
\hline$\gamma$-glutamyl Amino Acid & $\gamma$-glutamylglycine & HMDB11667 & 1.04 & 0.937 & 1.05 & 0.848 & 1.35 & 0.039 & 0.94 & 0.506 \\
\hline \multicolumn{11}{|l|}{ Xenobiotics } \\
\hline \multirow{2}{*}{ Benzoate } & 3-(2-hydroxyphenyl)propionate & HMDB33752 & 3.99 & 0.019 & 2.00 & 0.317 & 2.90 & 0.205 & 2.01 & 0.366 \\
\hline & 3-methoxycatechol sulfate & - & 2.95 & 0.021 & 2.97 & 0.083 & 1.24 & 0.499 & 0.91 & 0.642 \\
\hline Chemical & 6-hydroxyindole sulfate & - & 1.14 & 0.772 & 1.24 & 0.543 & 0.76 & 0.049 & 0.88 & 0.204 \\
\hline \multirow{2}{*}{ Drugs } & 4-hydroxycoumarin & - & 6.82 & 0.031 & 2.22 & 0.225 & 8.37 & 0.043 & 3.85 & 0.058 \\
\hline & Hydroquinone sulfate & HMDB02434 & 1.90 & 0.061 & 2.98 & 0.008 & 2.67 & 0.090 & 3.04 & 0.013 \\
\hline \multirow{7}{*}{ Phytochemicals } & 2-piperidinone ${ }^{1}$ & - & 1.21 & 0.384 & 1.09 & 0.763 & 0.70 & 0.005 & 0.58 & 0.000 \\
\hline & 4-allylphenol sulfate & - & 4.45 & 0.000 & 7.58 & 0.000 & 5.14 & 0.000 & 4.44 & 0.000 \\
\hline & $\beta$-guanidinopropanoate & HMDB13222 & 0.87 & 0.165 & 0.70 & 0.063 & 1.28 & 0.717 & 1.82 & 0.025 \\
\hline & Dihydroferulic acid & - & 3.56 & 0.025 & 3.71 & 0.010 & 2.19 & 0.203 & 2.32 & 0.207 \\
\hline & Saccharin & HMDB29723 & 1.06 & 0.486 & 1.09 & 0.845 & 1.40 & 0.010 & 1.74 & 0.045 \\
\hline & Tartarate $^{1}$ & HMDB00956 & 1.44 & 0.854 & 1.33 & 0.562 & 0.69 & 0.067 & 0.59 & 0.022 \\
\hline & Theanine & HMDB34365 & 43.91 & 0.014 & 6.53 & 0.336 & 7.81 & 0.618 & 5.39 & 0.729 \\
\hline Xanthine & Theobromine & HMDB02825 & 0.98 & 0.042 & 1.01 & 0.153 & 0.99 & 0.666 & 2.49 & 0.251 \\
\hline
\end{tabular}

GPC: Glycerophosphocholine; GPE: Glycerophosphoethanolamine; GPG: Glycerophosphoglycerol; GPI: Glycerophosphoinositol; CEHC: Carboxyethyl-hydroxychromans; HODE: Hydroxy-10(E),12(Z)-octadecadienoic acid; HMDB: Human metabolome database

${ }^{1}$ Metabolite also identified from the Rice Bran metabolome

*Values presented are fold-change of the mean relative abundance within control or rice bran group at 2 and 4 weeks compared to their baselines ( $p \leq 0.05)$. Statistically-significantly increased fold-changes are highlighted in red and statistically-significantly decreased fold-changes are highlighted in blue. Light blue and light red coloring represents metabolites and pathways that showed trends towards significance $(0.05 \leq \mathrm{p} \leq 0.10)$ 


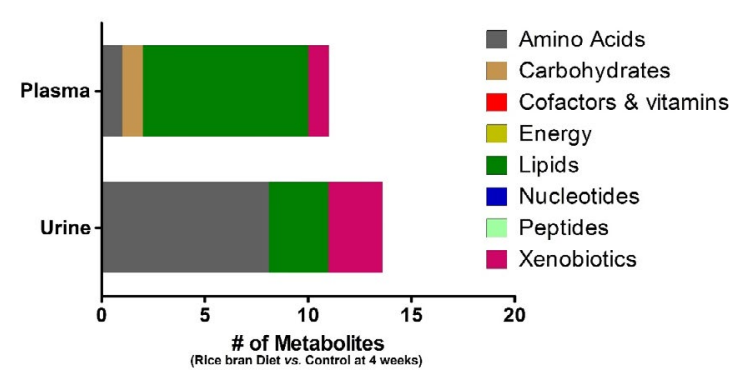

B

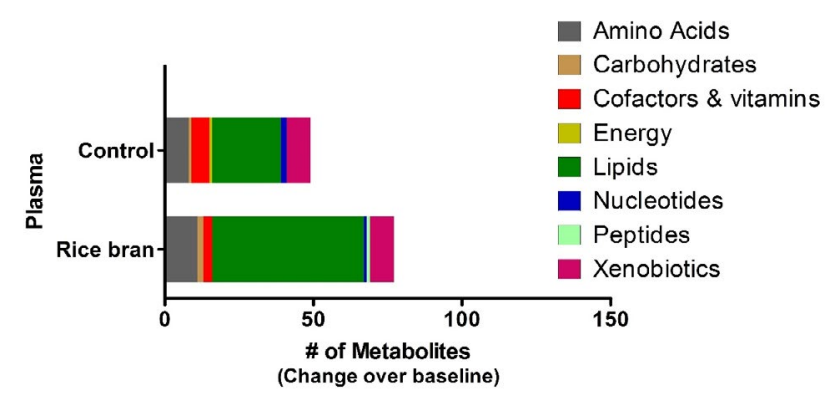

C

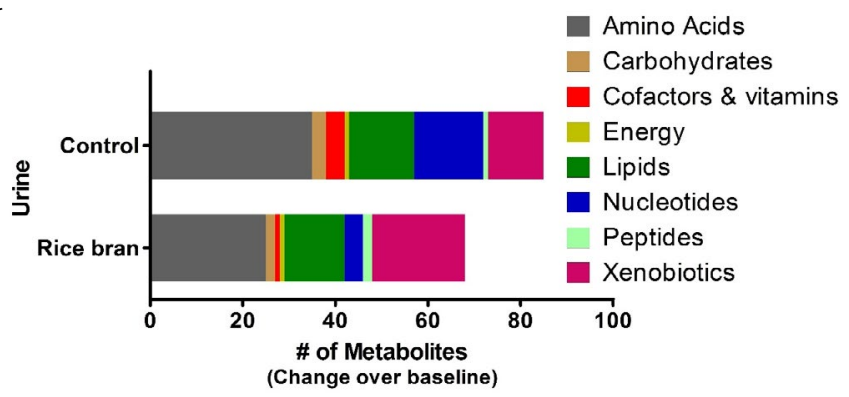

Figure 2. Chemical classes that were significantly modulated after 2 or 4 weeks of rice bran consumption when compared to control group or baseline. (A) Total number of metabolites with significant fold-differences in plasma and urine metabolome of CRC survivors at 4 weeks. (B) Total number of metabolites with significant fold-changes in plasma metabolome of CRC survivors at 2 and 4 weeks compared to baseline. (C) Total number of metabolites with significant fold-changes in urine metabolome of CRC survivors at 2 and 4 weeks compared to baseline. Significance was defined as metabolites with $p$-value $\leq 0.05$

intervention compared to baseline. These metabolite changes over time are also illustrated in Figure 2B $(p \leq 0.05)$. Forty-nine metabolites in the control group and 77 metabolites in the rice bran group showed significant fold-changes compared to baseline. In the control group, the significantly modulated metabolites were 23 lipids, 8 amino acids, 8 xenobiotics, 6 cofactors \& vitamins, 2 nucleotides, 1 carbohydrate, and 1 energy metabolite. In the rice bran group, the significantly modulated metabolites were 51 lipids, 11 amino acids, 8 xenobiotics, 3 cofactors \& vitamins, 2 carbohydrates, 1 nucleotide, and 1 peptide at 2 or 4 weeks compared to baseline. For all participants, data are presented as the mean fold-change at 2 or 4 weeks compared to baseline. Among the 77 metabolites in the rice bran group that changed at 2 or 4 weeks post intervention compared to baseline, 7 metabolites were also significantly different from between-group analysis (i.e. at 4 weeks compared to the control group). BCG was a metabolite with increased fold difference between groups and significant for changes over time ( BCG 1.67-fold), while decreased abundance was measured for linoleoylcholine (0.37-fold), oleoylcholine (0.38-fold), DGLA (0.87-fold), arachidonoylcholine (0.36-fold), dihomo-linolenoylcholine (0.33-fold), and docosahexaenoylcholine (0.36-fold) at 4 weeks compared to baseline.

\section{Dietary modulation of urine metabolome in CRC survivors}

Table 3 lists 14 urine metabolites with significant fold-differences between the rice bran and control participants at 4 weeks $(p \leq 0.05)$. These urine metabolites were 8 amino acids, 3 xenobiotics, and 3 lipids and are visualized in Figure 2A. Among the 14 urine metabolites with significant fold-differences in the rice bran group at 4 weeks compared to control, 6 metabolites were also identified from the rice bran food metabolome, namely $\mathrm{N}$-acetylisoleucine, $\mathrm{N}$-acetylvaline, $\mathrm{N}$-formylphenylalanine, phenylalanine, O-sulfo-L-tyrosine, and 3-(4-hydroxyphenyl) (footnote ${ }^{1}$ in Table 3). The 13 metabolites with significantly increased fold-differences included $\mathrm{N}$-acetylisoleucine (1.56-fold), $\mathrm{N}$-acetylvaline (1.44-fold), $\mathrm{N}$-methylleucine (3.37-fold), 3-(3-hydroxyphenyl)propionate (5.74-fold), $\mathrm{N}$-formylphenylalanine (2.19-fold), phenylalanine (1.41-fold), pro-hydroxy-pro (1.59-fold), $\mathrm{N}$-octanoylglycine (2.92-fold), androstenediol $(3 \beta, 17 \beta)$ monosulfate (5.34-fold), dehydroisoandrosterone sulfate (DHEA-S) (4.49-fold), 4-vinylphenol sulfate (2.61-fold), O-sulfo-L-tyrosine (1.37-fold), and theophylline (2.81-fold). The only urine metabolite with significantly decreased fold-difference was 3-(4-hydroxyphenyl)lactate (0.61-fold).

The urine metabolites that had significant fold-changes at 2 or 4 weeks compared to baseline are listed in Table 4 and visualized in Figure $2 \mathrm{C}(p \leq 0.05)$. These included 85 metabolites in the control group and 68 metabolites in the rice bran group. For the control, there were changes in 35 urine amino acids, 15 nucleotides, 14 lipids, 12 xenobiotics, 4 cofactors \& vitamins, 3 carbohydrates, 1 energy metabolite, and 1 peptide. Metabolites that were significantly modulated by rice bran

Table 3. Rice bran consumption showed different urine metabolites at 4 weeks compared to control group

\begin{tabular}{|c|c|c|c|}
\hline \multirow[b]{2}{*}{ Metabolite } & \multirow[b]{2}{*}{ HMDB } & \multicolumn{2}{|c|}{ Fold-difference* } \\
\hline & & Rice Bran & $p$-value \\
\hline \multicolumn{4}{|l|}{ Amino Acid } \\
\hline 3-(3-hydroxyphenyl)propionate & HMDB00375 & 5.74 & 0.038 \\
\hline 3-(4-hydroxyphenyl)lactate ${ }^{1}$ & HMDB00755 & 0.61 & 0.032 \\
\hline Indolepropionate & HMDB02302 & 0.37 & 0.054 \\
\hline Isovalerylglutamine & - & 0.68 & 0.097 \\
\hline $\mathrm{N}$-acetylisoleucine ${ }^{1}$ & - & 1.56 & 0.027 \\
\hline $\mathrm{N}$-acetylvaline ${ }^{1}$ & HMDB11757 & 1.44 & 0.039 \\
\hline N-formylphenylalanine ${ }^{1}$ & - & 2.19 & 0.030 \\
\hline N-methylleucine & - & 3.37 & 0.042 \\
\hline Phenylalanine $^{1}$ & HMDB00159 & 1.41 & 0.037 \\
\hline Pro-hydroxy-pro & HMDB06695 & 1.59 & 0.015 \\
\hline Pyroglutamine $^{1}$ & - & 1.48 & 0.056 \\
\hline \multicolumn{4}{|l|}{ Lipid } \\
\hline N-octanoylglycine & HMDB00832 & 2.92 & 0.028 \\
\hline Androstenediol $(3 \beta, 17 \beta)$ monosulfate & HMDB03818 & 5.34 & 0.016 \\
\hline Dehydroisoandrosterone sulfate (DHEA-S) & HMDB01032 & 4.49 & 0.033 \\
\hline \multicolumn{4}{|l|}{ Xenobiotics } \\
\hline 4-vinylphenol sulfate & & 2.61 & 0.028 \\
\hline O-sulfo-L-tyrosine ${ }^{1}$ & - & 1.37 & 0.019 \\
\hline Theophylline & HMDB01889 & 2.81 & 0.040 \\
\hline
\end{tabular}

HMDB: Human metabolome database

${ }^{1}$ Metabolite also identified from the rice bran metabolome

$*$ Values presented are fold-difference of the mean relative abundance between rice bran group compared to control at 4 weeks $(p \leq 0.05)$. Statistically-significantly increased folddifferences are highlighted in red and statistically-significantly decreased fold-differences are highlighted in blue. Light blue and light red coloring represent metabolites and pathways that showed trends towards significance $(0.05 \leq \mathrm{p} \leq 0.10)$ 
Table 4. Modulated urine metabolites following control or rice bran consumption at 2 and 4 weeks post intervention when compared to baseline

\begin{tabular}{|c|c|c|c|c|c|c|c|c|c|c|}
\hline & \multirow{3}{*}{ Metabolite } & \multirow{3}{*}{ HMDB } & \multicolumn{8}{|c|}{ Fold-change* } \\
\hline & & & \multicolumn{4}{|c|}{ Control group } & \multicolumn{4}{|c|}{ Rice bran group } \\
\hline & & & $\begin{array}{l}2 \mathrm{wk} / \\
0 \mathrm{wk}\end{array}$ & p-value & $\begin{array}{l}4 \mathrm{wk} / \\
0 \mathrm{wk}\end{array}$ & p-value & $\begin{array}{c}2 \mathrm{wk} / \\
0 \mathrm{wk}\end{array}$ & p-value & $\begin{array}{c}4 w k / \\
0 w k\end{array}$ & p-value \\
\hline \multicolumn{11}{|l|}{ Amino Acid } \\
\hline \multirow{4}{*}{ Alanine and Aspartate } & Alanine $^{1}$ & HMDB00161 & 0.95 & 0.502 & 0.99 & 0.951 & 0.80 & 0.016 & 0.74 & 0.002 \\
\hline & Asparagine $^{1}$ & HMDB00168 & 0.96 & 0.664 & 0.95 & 0.543 & 0.76 & 0.008 & 0.73 & 0.003 \\
\hline & $\mathrm{N}$-acetylalanine & HMDB00766 & 0.72 & 0.049 & 0.97 & 0.850 & 0.73 & 0.082 & 0.68 & 0.032 \\
\hline & $\mathrm{N}$-acetylaspartate (NAA) ${ }^{1}$ & HMDB00812 & 0.97 & 0.618 & 0.88 & 0.072 & 1.24 & 0.007 & 0.94 & 0.422 \\
\hline \multirow{2}{*}{ Glutamate } & Carboxyethyl-GABA $^{1}$ & HMDB02201 & 0.82 & 0.157 & 0.72 & 0.018 & 1.14 & 0.377 & 0.83 & 0.219 \\
\hline & N-methyl-GABA & - & 0.57 & 0.049 & 0.51 & 0.020 & 0.62 & 0.133 & 0.79 & 0.445 \\
\hline Glutathione & 5-oxoproline ${ }^{1}$ & HMDB00267 & 0.89 & 0.138 & 0.89 & 0.153 & 1.24 & 0.016 & 0.98 & 0.805 \\
\hline Glycine, Serine and Threonine & $\mathrm{N}^{-a c e t y l s e r i n e}{ }^{1}$ & HMDB02931 & 0.99 & 0.923 & 0.90 & 0.319 & 0.72 & 0.007 & 0.74 & 0.015 \\
\hline Guanidino and Acetamido & 1-methylguanidine & HMDB01522 & 0.94 & 0.604 & 0.72 & 0.011 & 1.29 & 0.072 & 1.00 & 0.972 \\
\hline \multirow{3}{*}{ Histidine } & 1-methylhistamine & HMDB00898 & 0.88 & 0.320 & 0.77 & 0.040 & 0.97 & 0.808 & 1.03 & 0.849 \\
\hline & 4-imidazoleacetate ${ }^{1}$ & HMDB02024 & 1.25 & 0.042 & 1.13 & 0.268 & 1.07 & 0.569 & 1.10 & 0.414 \\
\hline & $\mathrm{N}$-acetyl-1-methylhistidine & & 0.78 & 0.072 & 0.62 & 0.001 & 0.90 & 0.494 & 0.86 & 0.312 \\
\hline \multirow{7}{*}{ Leucine, Isoleucine and Valine } & 3-methyl-2-oxovalerate ${ }^{1}$ & HMDB03736 & 0.45 & 0.047 & 0.62 & 0.221 & 0.69 & 0.400 & 0.57 & 0.202 \\
\hline & 3-methylcrotonylglycine & HMDB00459 & 1.40 & 0.031 & 1.41 & 0.026 & 1.35 & 0.077 & 1.19 & 0.303 \\
\hline & Isobutyrylcarnitine (C4) & HMDB00736 & 1.47 & 0.009 & 1.29 & 0.076 & 1.16 & 0.330 & 1.20 & 0.248 \\
\hline & Isobutyrylglycine $^{1}$ & HMDB00730 & 1.56 & 0.004 & 1.41 & 0.023 & 1.19 & 0.291 & 1.27 & 0.140 \\
\hline & Isovalerylglutamine & - & 1.18 & 0.250 & 1.33 & 0.047 & 0.86 & 0.346 & 0.80 & 0.151 \\
\hline & Isovalerylglycine $^{1}$ & HMDB00678 & 1.37 & 0.043 & 1.37 & 0.041 & 1.07 & 0.670 & 1.01 & 0.976 \\
\hline & Methylsuccinoylcarnitine & - & 0.97 & 0.761 & 0.85 & 0.102 & 1.28 & 0.031 & 1.17 & 0.175 \\
\hline \multirow{3}{*}{ Lysine } & Glutarate (pentanedioate) $^{1}$ & HMDB00661 & 1.21 & 0.145 & 1.06 & 0.622 & 1.36 & 0.033 & 1.11 & 0.440 \\
\hline & N2,N6-diacetyllysine & - & 0.95 & 0.567 & 0.82 & 0.025 & 1.01 & 0.891 & 0.94 & 0.493 \\
\hline & N6,N6,N6-trimethyllysine & HMDB01325 & 0.93 & 0.561 & 0.64 & 0.001 & 1.00 & 0.977 & 1.18 & 0.229 \\
\hline \multirow{5}{*}{ Methionine, Cysteine, SAM and Taurine } & Cystathionine $^{1}$ & HMDB00099 & 1.29 & 0.136 & 1.64 & 0.004 & 1.00 & 0.998 & 1.47 & 0.042 \\
\hline & Methionine $^{1}$ & HMDB00696 & 1.09 & 0.693 & 1.01 & 0.951 & 0.80 & 0.373 & 0.58 & 0.032 \\
\hline & $\mathrm{N}$-methyltaurine & - & 2.70 & 0.019 & 2.05 & 0.085 & 0.90 & 0.807 & 0.93 & 0.871 \\
\hline & S-methylcysteine sulfoxide & - & 1.27 & 0.161 & 1.58 & 0.009 & 1.36 & 0.106 & 1.17 & 0.403 \\
\hline & Taurocyamine & HMDB03584 & 0.95 & 0.812 & 1.04 & 0.842 & 0.79 & 0.326 & 0.57 & 0.019 \\
\hline \multirow{14}{*}{ Phenylalanine and Tyrosine } & 3-(3-hydroxyphenyl)propionate sulfate & - & 0.78 & 0.289 & 0.69 & 0.119 & 1.91 & 0.015 & 1.49 & 0.128 \\
\hline & 3-(4-hydroxyphenyl)lactate ${ }^{1}$ & HMDB00755 & 1.14 & 0.355 & 1.52 & 0.004 & 1.05 & 0.751 & 0.86 & 0.348 \\
\hline & 3,4-dihydroxyphenylacetate & HMDB01336 & 0.82 & 0.461 & 0.53 & 0.022 & 1.44 & 0.215 & 1.16 & 0.623 \\
\hline & 3-hydroxyphenylacetate sulfate & - & 0.90 & 0.645 & 0.65 & 0.050 & 1.72 & 0.029 & 1.44 & 0.136 \\
\hline & 4-hydroxycinnamate sulfate & - & 1.38 & 0.265 & 1.82 & 0.040 & 0.90 & 0.749 & 0.97 & 0.922 \\
\hline & 4-hydroxyphenylacetatoylcarnitine & - & 1.24 & 0.242 & 1.48 & 0.035 & 1.19 & 0.382 & 1.16 & 0.468 \\
\hline & 5-bromotryptophan & - & 0.84 & 0.119 & 0.80 & 0.049 & 1.23 & 0.098 & 1.16 & 0.229 \\
\hline & Catechol glucuronide & - & 0.83 & 0.531 & 1.00 & 0.990 & 2.93 & 0.002 & 1.37 & 0.336 \\
\hline & Homovanillate (HVA) & HMDB00118 & 1.01 & 0.959 & 0.89 & 0.313 & 1.31 & 0.046 & 1.05 & 0.687 \\
\hline & Homovanillate sulfate & HMDB11719 & 0.90 & 0.438 & 0.76 & 0.054 & 1.44 & 0.018 & 1.04 & 0.801 \\
\hline & Phenol sulfate & HMDB60015 & 1.08 & 0.610 & 0.96 & 0.787 & 2.64 & 0.000 & 2.31 & 0.000 \\
\hline & Phenyllactate (PLA) ${ }^{1}$ & HMDB00779 & 1.16 & 0.473 & 1.75 & 0.009 & 0.75 & 0.215 & 0.79 & 0.306 \\
\hline & Vanillactate & HMDB00913 & 1.52 & 0.044 & 1.32 & 0.174 & 1.72 & 0.018 & 1.07 & 0.777 \\
\hline & Vanillylmandelate (VMA) & HMDB00291 & 0.99 & 0.889 & 0.87 & 0.108 & 1.29 & 0.006 & 1.02 & 0.847 \\
\hline \multirow{3}{*}{ Polyamine } & Acisoga & - & 0.90 & 0.344 & 0.94 & 0.581 & 1.29 & 0.047 & 1.15 & 0.260 \\
\hline & $\mathrm{N}(4)$-acetylspermidine & - & 0.88 & 0.222 & 0.76 & 0.012 & 0.98 & 0.889 & 0.91 & 0.432 \\
\hline & Spermidine $^{1}$ & HMDB01257 & 1.14 & 0.592 & 1.05 & 0.835 & 1.80 & 0.038 & 1.58 & 0.102 \\
\hline \multirow{7}{*}{ Tryptophan } & 3-hydroxyanthranilate & HMDB01476 & 2.09 & 0.003 & 1.64 & 0.037 & 0.97 & 0.918 & 0.93 & 0.770 \\
\hline & C-glycosyltryptophan & - & 0.95 & 0.612 & 0.83 & 0.096 & 1.30 & 0.037 & 1.06 & 0.606 \\
\hline & Indolelactate & HMDB00671 & 1.25 & 0.027 & 1.08 & 0.436 & 1.05 & 0.665 & 0.90 & 0.333 \\
\hline & Indolepropionate & HMDB02302 & 1.51 & 0.346 & 3.05 & 0.013 & 1.65 & 0.295 & 1.36 & 0.521 \\
\hline & Kynurenate $^{1}$ & HMDB00715 & 1.31 & 0.002 & 1.07 & 0.407 & 1.24 & 0.027 & 1.03 & 0.715 \\
\hline & Tryptophan betaine & HMDB61115 & 0.94 & 0.730 & 1.25 & 0.189 & 0.68 & 0.039 & 0.89 & 0.521 \\
\hline & Xanthurenate $^{1}$ & HMDB00881 & 1.37 & 0.005 & 1.16 & 0.180 & 1.37 & 0.011 & 1.17 & 0.201 \\
\hline & Argininate & HMDB03148 & 1.29 & 0.034 & 1.09 & 0.470 & 0.95 & 0.675 & 0.94 & 0.640 \\
\hline Urea cycle; Arginine and Proline & Methylurea & - & 1.36 & 0.036 & 1.12 & 0.424 & 1.11 & 0.492 & 0.82 & 0.210 \\
\hline & Pro-hydroxy-pro & HMDB06695 & 0.86 & 0.208 & 0.59 & 0.000 & 1.17 & 0.229 & 1.11 & 0.422 \\
\hline
\end{tabular}




\begin{tabular}{|c|c|c|c|c|c|c|c|c|c|c|}
\hline & \multirow{3}{*}{ Metabolite } & \multirow{3}{*}{ HMDB } & \multicolumn{8}{|c|}{ Fold-change* } \\
\hline & & & \multicolumn{4}{|c|}{ Control group } & \multicolumn{4}{|c|}{ Rice bran group } \\
\hline & & & $\begin{array}{l}2 \mathrm{wk} / \\
0 \mathrm{wk}\end{array}$ & p-value & $\begin{array}{c}4 \mathrm{wk} / \\
0 \mathrm{wk}\end{array}$ & p-value & $\begin{array}{c}2 \mathrm{wk} / \\
0 \mathrm{wk}\end{array}$ & p-value & $\begin{array}{c}4 \mathrm{wk} / \\
0 \mathrm{wk}\end{array}$ & p-value \\
\hline \multicolumn{11}{|l|}{ Carbohydrate } \\
\hline Advanced Glycation End-product & N6-carboxymethyllysine ${ }^{1}$ & - & 1.45 & 0.019 & 1.07 & 0.649 & 1.24 & 0.211 & 0.99 & 0.960 \\
\hline \multirow{2}{*}{ Aminosugar } & 6-sialyl-N-acetyllactosamine & HMDB06584 & 0.95 & 0.703 & 0.75 & 0.039 & 1.14 & 0.400 & 1.05 & 0.724 \\
\hline & $\mathrm{N}$-acetylneuraminate & HMDB00230 & 0.93 & 0.501 & 0.83 & 0.065 & 1.32 & 0.016 & 1.08 & 0.502 \\
\hline Glycolysis, Gluconeogenesis, and Pyruvate & Lactate $^{1}$ & HMDB00190 & 1.07 & 0.714 & 1.15 & 0.437 & 0.68 & 0.064 & 0.64 & 0.030 \\
\hline Pentose & Ribonate $^{1}$ & HMDB00867 & 1.52 & 0.034 & 1.58 & 0.022 & 1.49 & 0.068 & 1.21 & 0.381 \\
\hline \multicolumn{11}{|l|}{ Cofactors \& vitamins } \\
\hline Hemoglobin and Porphyrin & L-urobilin & HMDB04159 & 0.49 & 0.032 & 0.75 & 0.369 & 1.09 & 0.808 & 1.33 & 0.429 \\
\hline Nicotinate and Nicotinamide & Nicotinate $^{1}$ & HMDB01488 & 1.66 & 0.073 & 0.91 & 0.733 & 1.10 & 0.764 & 0.54 & 0.050 \\
\hline \multirow{3}{*}{ Pterin } & Pterin $^{1}$ & HMDB00802 & 0.79 & 0.154 & 0.59 & 0.002 & 1.15 & 0.438 & 1.03 & 0.852 \\
\hline & Sepiapterin & HMDB00238 & 0.86 & 0.632 & 0.48 & 0.028 & 1.32 & 0.440 & 0.93 & 0.830 \\
\hline & Xanthopterin & - & 0.58 & 0.015 & 0.54 & 0.007 & 0.88 & 0.609 & 0.77 & 0.275 \\
\hline \multicolumn{11}{|l|}{ Energy } \\
\hline \multirow{2}{*}{ TCA Cycle } & 2-methylcitrate & HMDB00379 & 0.98 & 0.872 & 0.90 & 0.272 & 1.28 & 0.029 & 1.03 & 0.764 \\
\hline & Citraconate/glutaconate & - & 0.54 & 0.043 & 0.49 & 0.018 & 1.49 & 0.219 & 1.02 & 0.942 \\
\hline \multicolumn{11}{|l|}{ Lipid } \\
\hline \multirow{3}{*}{ Fatty Acid (Acyl Glutamine) } & Heptanoylglutamine & - & 0.60 & 0.004 & 0.65 & 0.017 & 1.33 & 0.136 & 1.20 & 0.343 \\
\hline & Hexanoylglutamine & - & 0.63 & 0.011 & 0.70 & 0.043 & 1.40 & 0.086 & 1.20 & 0.351 \\
\hline & $\mathrm{N}$-octanoylglutamine & - & 0.68 & 0.038 & 0.72 & 0.071 & 1.63 & 0.018 & 1.42 & 0.085 \\
\hline \multirow{5}{*}{ Fatty Acid (Acyl Carnitine) } & Adipoylcarnitine (C6-DC) & - & 0.80 & 0.175 & 0.87 & 0.381 & 1.84 & 0.001 & 1.43 & 0.051 \\
\hline & Laurylcarnitine (C12) & HMDB02250 & 0.92 & 0.690 & 0.59 & 0.021 & 1.23 & 0.391 & 1.21 & 0.423 \\
\hline & Myristoleoylcarnitine (C14:1) & - & 0.92 & 0.734 & 0.58 & 0.037 & 1.47 & 0.179 & 1.20 & 0.531 \\
\hline & $\begin{array}{l}\text { Pimeloylcarnitine/3-methyladipoylcarnitine } \\
\text { (C7-DC) }\end{array}$ & - & 1.04 & 0.762 & 1.05 & 0.668 & 1.56 & 0.002 & 1.13 & 0.380 \\
\hline & Suberoylcarnitine (C8-DC) & - & 0.63 & 0.009 & 0.68 & 0.027 & 1.53 & 0.025 & 1.14 & 0.474 \\
\hline Fatty Acid (Acyl Glycine) & Hexanoylglycine $^{1}$ & HMDB00701 & 0.94 & 0.675 & 0.88 & 0.408 & 1.54 & 0.013 & 1.34 & 0.086 \\
\hline & 3-methyladipate ${ }^{1}$ & HMDB00555 & 0.92 & 0.638 & 0.86 & 0.401 & 1.84 & 0.003 & 1.08 & 0.683 \\
\hline Fatty Acid, Dicarboxylate & 4-octenedioate & HMDB04982 & 0.76 & 0.059 & 0.73 & 0.033 & 1.39 & 0.041 & 1.12 & 0.457 \\
\hline & Dimethylmalonic acid ${ }^{1}$ & HMDB02001 & 1.33 & 0.025 & 1.21 & 0.129 & 1.22 & 0.150 & 1.22 & 0.155 \\
\hline Fattv Acid Monohydroxy & 3-hydroxysebacate & HMDB00350 & 0.51 & 0.005 & 0.62 & 0.045 & 1.16 & 0.567 & 1.09 & 0.726 \\
\hline Fatty Acı, Monohydroxy & 3-hydroxysuberate ${ }^{1}$ & HMDB00325 & 0.59 & 0.014 & 0.54 & 0.004 & 1.38 & 0.158 & 1.14 & 0.577 \\
\hline Ketone Bodies & 3-hydroxybutyrate (BHBA) ${ }^{1}$ & HMDB00357 & 0.51 & 0.021 & 0.60 & 0.078 & 0.89 & 0.719 & 0.92 & 0.803 \\
\hline Mevalonate & Mevalonate & HMDB00227 & 0.61 & 0.039 & 0.70 & 0.128 & 0.72 & 0.207 & 0.63 & 0.077 \\
\hline Phosnholinid & Glycerophosphoethanolamine $^{1}$ & HMDB00114 & 0.93 & 0.483 & 0.77 & 0.015 & 1.11 & 0.380 & 0.94 & 0.605 \\
\hline Pliosprionpia & Trimethylamine N-oxide & HMDB00925 & 1.31 & 0.046 & 1.19 & 0.185 & 1.43 & 0.016 & 1.14 & 0.362 \\
\hline Primary Bile Acid & Cholate & HMDB00619 & 0.77 & 0.519 & 1.00 & 0.995 & 0.25 & 0.003 & 0.52 & 0.156 \\
\hline & Deoxycholic acid sulfate & - & 0.83 & 0.643 & 0.79 & 0.562 & 0.37 & 0.027 & 0.37 & 0.024 \\
\hline 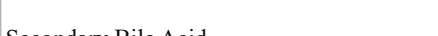 & Glycolithocholate sulfate & HMDB02639 & 0.79 & 0.390 & 0.70 & 0.194 & 1.97 & 0.029 & 1.60 & 0.125 \\
\hline Secondary Bile Acid & Taurocholenate sulfate & - & 0.76 & 0.223 & 0.85 & 0.484 & 1.87 & 0.015 & 1.43 & 0.161 \\
\hline & Taurolithocholate 3-sulfate & HMDB02580 & 0.60 & 0.106 & 0.76 & 0.383 & 2.25 & 0.020 & 1.55 & 0.202 \\
\hline Nucleotide & & & & & & & & & & \\
\hline & Urate $^{1}$ & HMDB00289 & 0.82 & 0.116 & 0.72 & 0.013 & 0.93 & 0.580 & 0.89 & 0.388 \\
\hline Purine, (Hypo)Xanthine/Inosine containing & Xanthine $^{1}$ & HMDB00292 & 0.68 & 0.020 & 0.70 & 0.027 & 1.13 & 0.480 & 0.91 & 0.578 \\
\hline & Xanthosine $^{1}$ & HMDB00299 & 0.82 & 0.036 & 0.80 & 0.022 & 1.19 & 0.105 & 0.94 & 0.569 \\
\hline Purine, Adenine containing & $\begin{array}{l}\text { Adenosine-3',5'-cyclic monophosphate } \\
\text { (cAMP) }\end{array}$ & HMDB00058 & 0.88 & 0.108 & 0.81 & 0.011 & 1.27 & 0.010 & 1.07 & 0.434 \\
\hline & N1-methyladenosine & HMDB03331 & 0.84 & 0.086 & 0.72 & 0.002 & 0.97 & 0.796 & 0.89 & 0.274 \\
\hline & $2^{\prime}$-deoxyguanosine ${ }^{1}$ & HMDB00085 & 0.71 & 0.012 & 0.82 & 0.140 & 1.31 & 0.065 & 0.93 & 0.603 \\
\hline & 7-methylguanine ${ }^{1}$ & HMDB00897 & 0.85 & 0.188 & 0.76 & 0.028 & 1.07 & 0.598 & 0.92 & 0.519 \\
\hline & Guanosine $^{1}$ & HMDB00133 & 0.76 & 0.350 & 0.47 & 0.012 & 1.30 & 0.418 & 0.71 & 0.292 \\
\hline Purine, Guanine containing & $\begin{array}{l}\text { Guanosine-3',5'-cyclic monophosphate } \\
\text { (cGMP) }\end{array}$ & HMDB01314 & 0.68 & 0.002 & 0.74 & 0.016 & 1.02 & 0.900 & 0.92 & 0.552 \\
\hline & N1-methylguanosine' & HMDB01563 & 0.87 & 0.513 & 0.63 & 0.031 & 1.13 & 0.601 & 0.95 & 0.817 \\
\hline & N2-methylguanosine & HMDB05862 & 0.82 & 0.132 & 0.76 & 0.031 & 1.09 & 0.520 & 0.99 & 0.931 \\
\hline Pyrimidine, Cytidine containing & Cytidine $^{1}$ & HMDB00089 & 0.85 & 0.299 & 0.71 & 0.029 & 1.00 & 0.999 & 0.84 & 0.315 \\
\hline & N4-acetylcytidine & HMDB05923 & 0.86 & 0.158 & 0.86 & 0.150 & 1.29 & 0.029 & 0.99 & 0.921 \\
\hline & 3-ureidopropionate & HMDB00026 & 0.89 & 0.340 & 0.94 & 0.611 & 1.42 & 0.015 & 0.99 & 0.944 \\
\hline D. i. & 4-ureidobutyrate ${ }^{1}$ & - & 1.48 & 0.027 & 1.23 & 0.242 & 1.07 & 0.720 & 1.34 & 0.131 \\
\hline Pyrimıdine, Uracil containing & Pseudouridine $^{1}$ & HMDB00767 & 0.87 & 0.061 & 0.84 & 0.017 & 1.21 & 0.019 & 1.04 & 0.623 \\
\hline & Uracil $^{1}$ & HMDB00300 & 0.86 & 0.259 & 0.76 & 0.045 & 1.03 & 0.846 & 0.86 & 0.314 \\
\hline
\end{tabular}




\begin{tabular}{|c|c|c|c|c|c|c|c|c|c|c|}
\hline & \multirow{3}{*}{ Metabolite } & \multirow{3}{*}{ HMDB } & \multicolumn{8}{|c|}{ Fold-change* } \\
\hline & & & \multicolumn{4}{|c|}{ Control group } & \multicolumn{4}{|c|}{ Rice bran group } \\
\hline & & & $\begin{array}{l}2 \mathrm{wk} / \\
0 \mathrm{wk}\end{array}$ & p-value & $\begin{array}{c}4 \mathrm{wk} / \\
0 \mathrm{wk}\end{array}$ & p-value & $\begin{array}{c}2 \mathrm{wk} / \\
0 \mathrm{wk}\end{array}$ & p-value & $\begin{array}{l}4 \mathrm{wk} / \\
0 \mathrm{wk}\end{array}$ & p-value \\
\hline \multicolumn{11}{|l|}{ Peptide } \\
\hline Acetylated Peptides & Phenylacetylhistidine & - & 1.35 & 0.042 & 1.33 & 0.054 & 1.05 & 0.771 & 1.08 & 0.620 \\
\hline \multirow{2}{*}{$\gamma$-glutamyl Amino Acid } & $\gamma$-glutamylglutamine ${ }^{1}$ & HMDB11738 & 0.77 & 0.231 & 1.43 & 0.099 & 0.54 & 0.012 & 0.72 & 0.162 \\
\hline & $\gamma$-glutamylglycine & HMDB11667 & 0.99 & 0.940 & 1.11 & 0.472 & 0.68 & 0.013 & 0.74 & 0.050 \\
\hline \multicolumn{11}{|l|}{ Xenobiotics } \\
\hline \multirow{8}{*}{ Benzoate } & 3-hydroxyhippurate & HMDB06116 & 0.78 & 0.321 & 0.76 & 0.273 & 2.28 & 0.004 & 1.74 & 0.047 \\
\hline & 3-methoxycatechol sulfate [1] & - & 1.75 & 0.015 & 1.19 & 0.442 & 1.15 & 0.562 & 1.03 & 0.891 \\
\hline & 3-methoxycatechol sulfate [2] & - & 1.46 & 0.191 & 1.02 & 0.949 & 2.42 & 0.007 & 1.96 & 0.036 \\
\hline & 4-hydroxymandelate & HMDB00822 & 1.25 & 0.127 & 1.37 & 0.035 & 1.20 & 0.264 & 1.17 & 0.323 \\
\hline & 4-methylcatechol sulfate & - & 0.88 & 0.466 & 0.84 & 0.351 & 1.69 & 0.011 & 1.42 & 0.086 \\
\hline & Catechol sulfate & HMDB59724 & 0.89 & 0.483 & 0.85 & 0.321 & 1.74 & 0.004 & 1.20 & 0.325 \\
\hline & Hippurate & HMDB00714 & 0.84 & 0.383 & 0.75 & 0.174 & 1.60 & 0.041 & 1.22 & 0.392 \\
\hline & O-methylcatechol sulfate & - & 0.90 & 0.421 & 0.86 & 0.235 & 1.58 & 0.002 & 1.09 & 0.528 \\
\hline \multirow{10}{*}{ Chemical } & 2-methoxyresorcinol sulfate & - & 2.10 & 0.021 & 1.70 & 0.094 & 1.27 & 0.484 & 1.09 & 0.802 \\
\hline & 3-acetylphenol & - & 0.90 & 0.758 & 0.68 & 0.280 & 2.20 & 0.049 & 1.89 & 0.107 \\
\hline & 3-acetylphenol sulfate & - & 0.79 & 0.280 & 0.96 & 0.838 & 1.82 & 0.016 & 1.21 & 0.430 \\
\hline & 3-hydroxypyridine sulfate & - & 0.58 & 0.102 & 0.60 & 0.122 & 2.08 & 0.049 & 1.20 & 0.612 \\
\hline & 4'-hydroxypropiophenone sulfate & - & 2.00 & 0.024 & 2.49 & 0.003 & 2.05 & 0.033 & 1.89 & 0.058 \\
\hline & 6-hydroxyindole sulfate & - & 1.01 & 0.959 & 1.39 & 0.035 & 0.91 & 0.560 & 0.98 & 0.895 \\
\hline & Benzoylcarnitine & - & 0.81 & 0.104 & 0.72 & 0.011 & 1.27 & 0.083 & 1.28 & 0.076 \\
\hline & Dimethyl sulfone & HMDB04983 & 1.23 & 0.285 & 1.68 & 0.010 & 1.37 & 0.147 & 1.43 & 0.099 \\
\hline & Lanthionine & - & 1.08 & 0.589 & 0.93 & 0.585 & 0.90 & 0.503 & 0.68 & 0.012 \\
\hline & O-sulfo-L-tyrosine ${ }^{1}$ & - & 0.91 & 0.236 & 0.81 & 0.012 & 1.18 & 0.063 & 1.04 & 0.674 \\
\hline Drug & Hydroquinone sulfate & HMDB02434 & 1.22 & 0.444 & 1.37 & 0.217 & 3.52 & 0.000 & 3.33 & 0.000 \\
\hline \multirow{10}{*}{ Phytochemicals } & 2,3-dihydroxypyridine & - & 0.67 & 0.237 & 0.54 & 0.075 & 3.12 & 0.003 & 1.49 & 0.285 \\
\hline & 3-hydroxycinnamate sulfate & - & 0.61 & 0.059 & 0.53 & 0.019 & 2.07 & 0.014 & 1.48 & 0.177 \\
\hline & 3-hydroxyindolin-2-one ${ }^{1}$ & - & 1.35 & 0.262 & 1.29 & 0.348 & 0.82 & 0.503 & 0.46 & 0.011 \\
\hline & 4-allylphenol sulfate & - & 3.95 & 0.000 & 5.09 & 0.000 & 4.85 & 0.000 & 3.86 & 0.000 \\
\hline & Enterolactone & - & 1.63 & 0.180 & 1.02 & 0.950 & 5.93 & 0.000 & 3.74 & 0.002 \\
\hline & Erythritol $^{1}$ & HMDB02994 & 0.90 & 0.578 & 0.92 & 0.625 & 1.54 & 0.034 & 0.87 & 0.496 \\
\hline & $\mathrm{N}$-(2-furoyl)glycine & HMDB00439 & 0.57 & 0.145 & 0.71 & 0.369 & 2.61 & 0.024 & 1.06 & 0.887 \\
\hline & Tartarate $^{1}$ & HMDB00956 & 0.43 & 0.322 & 0.42 & 0.306 & 0.15 & 0.044 & 0.15 & 0.050 \\
\hline & Theanine & HMDB34365 & 2.83 & 0.040 & 1.83 & 0.226 & 1.00 & 0.993 & 1.25 & 0.681 \\
\hline & Thymol sulfate & HMDB01878 & 4.24 & 0.012 & 6.06 & 0.002 & 1.47 & 0.531 & 0.91 & 0.882 \\
\hline
\end{tabular}

GABA: $\gamma$-aminobutyric acid; CEHC: Carboxyethyl-hydroxychromans; HMDB: Human metabolome database

${ }^{1}$ Metabolite also identified from the rice bran metabolome

*Values presented are fold-change of the mean relative abundance within control or rice bran group at 2 and 4 weeks compared to their baselines ( $\leq \leq 0.05$ ). Statistically-significantly increased fold-changes are highlighted in red and statistically-significantly decreased fold-changes are highlighted in blue. Light blue and light red coloring represent metabolites and pathways that showed trends towards significance $(0.05 \leq \mathrm{p} \leq 0.10)$

intake at 2 or 4 weeks post intervention compared to baseline included 25 amino acids, 20 xenobiotics, 13 lipids, 4 nucleotides, 2 carbohydrates, 2 peptides, 1 cofactor \& vitamin, and 1 energy metabolite. Among the 68 significantly modulated urine metabolites over time, 20 metabolites were identified from the rice bran food metabolome (footnote ${ }^{1}$ in Table 4). N-acetylaspartate (NAA) (1.24-fold), 5-oxoproline (1.24-fold), glutarate (pentanedioate) (1.36-fold), spermidine (1.8-fold), kynurenate (1.24-fold), xanthurenate (1.37-fold), hexanoylglycine (1.54-fold), 3-methyladipate (1.84-fold), pseudouridine (1.21-fold), and erythritol (1.54-fold) increased at 2 weeks. Cystathionine was the only compound increased at 4 weeks (1.47-fold). Alanine (0.8-fold), asparagine (0.76fold), and $\mathrm{N}$-acetylserine (0.72-fold), $\gamma$-glutamylglutamine (0.54-fold), tartarate $(0.15$-fold $)$ decreased at 2 weeks after intervention. Alanine (0.74-fold), asparagine (0.73-fold), and $\mathrm{N}$-acetylserine (0.74-fold) were decreased at 4 weeks followed by lactate $(0.64$-fold), nicotinate $(0.54$ fold), and 3-hydroxyindolin-2-one (0.46-fold).

\section{Metabolic pathways affected by rice bran intake in plasma}

Figure 3 visualizes the 11 plasma metabolites that were significantly different in the rice bran group compared to control at 4 weeks, including the pathway enrichment scores which take into consideration all metabolites identified in the plasma for that metabolic pathway (as described in methods). Lipid metabolic pathways modulated following rice bran consumption included acyl choline fatty acids, $(\mathrm{PES}=42.7)$, acyl carnitine fatty acids (PES=3.3), and phospholipids ( $\mathrm{PES}=4.6$ ). Additional pathways affected by rice bran consumption were glutamate (PES=7.1), carbohydrate (fructose, mannose, and galactose) (PES=14.2), and xenobiotic (benzoate) (PES=3.6).

\section{Metabolic pathways affected by rice bran intake in urine}

Figure 4 visualizes the 14 urine metabolites that were significantly different in the rice bran group compared to control at 4 weeks with the PES for each metabolic pathway involved. The amino acid metabolic 

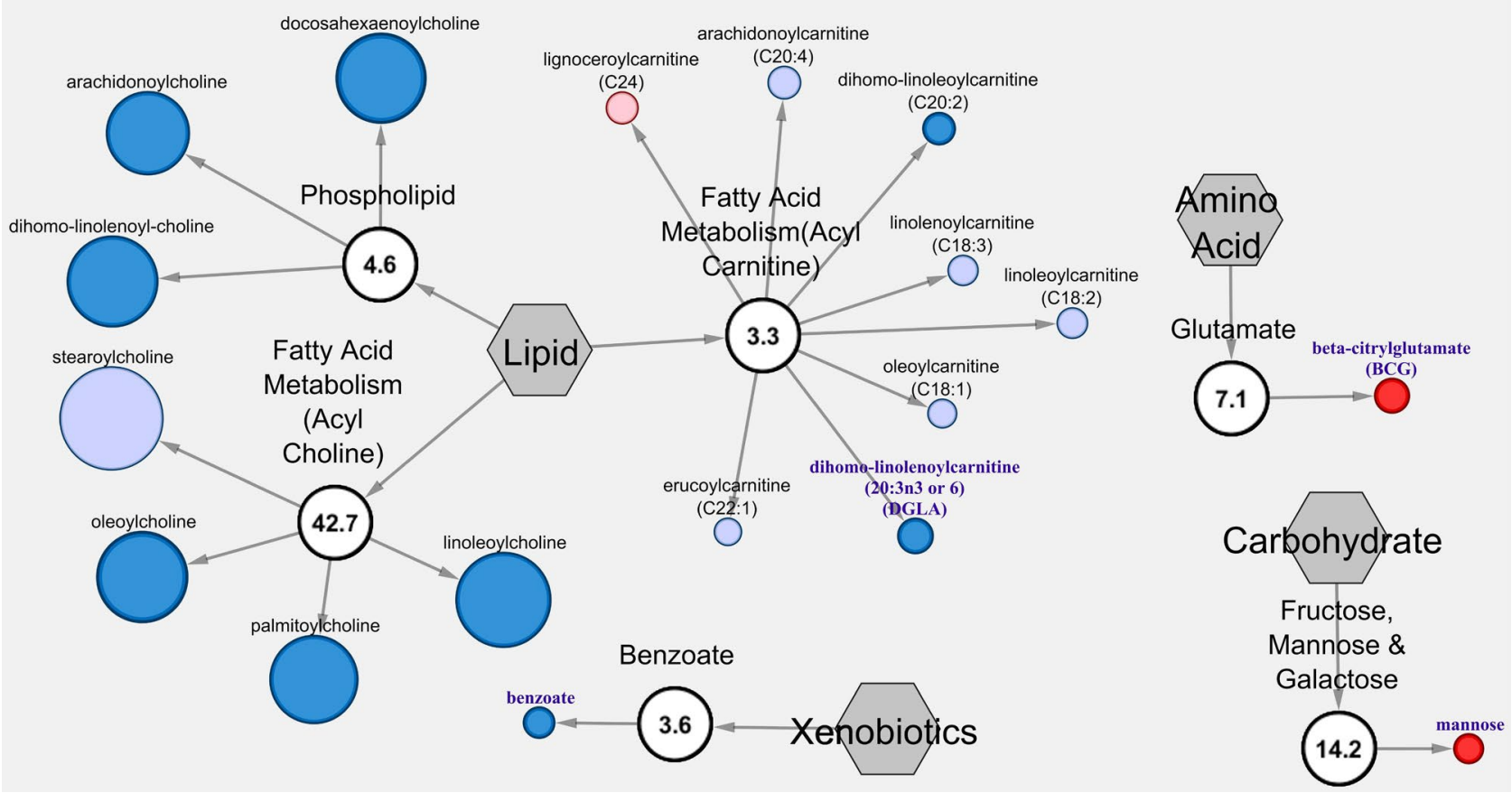

Figure 3. Plasma metabolic pathway visualization of rice bran group compared to control at 4 weeks. There were 6 metabolic pathway enrichment scores showing metabolites that are significantly different between the rice bran group and control at 4 weeks. Each metabolite was represented by a closed, colored node extending from a metabolic pathway node, which connects to a chemical class hexagonal node. A red node represents metabolites with significantly higher expression in rice bran group at 4 weeks compared to control group ( $p \leq 0 \cdot 05$ ). A dark blue node represents metabolites with significantly lower expression in rice bran group at 4 weeks when compared to control group. Nodes colored pink or light blue represent metabolites trending towards significance with higher expression in rice bran group at 4 weeks and lower expression at 4 weeks, respectively $(0 \cdot 05 \leq p \leq 0 \cdot 1)$. Node size is proportional to fold-difference magnitude at week 4 for rice bran group compared to control group. Bolded metabolites indicate previously reported evidence for cancer chemoprevention.

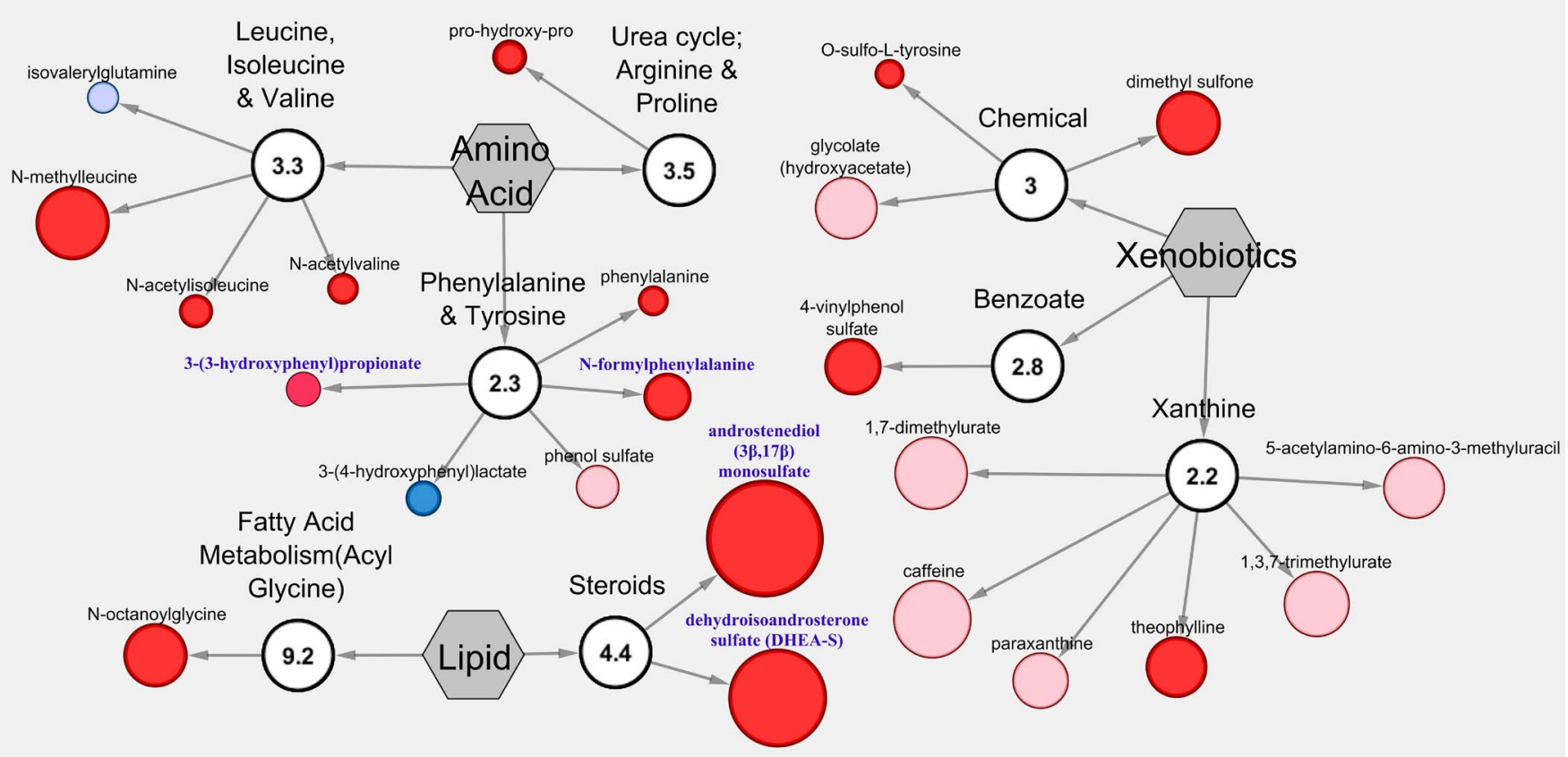

Figure 4. Urine metabolic pathway visualization of rice bran group compared to control at 4 weeks. There were 8 metabolic pathway enrichment scores that show metabolites that are significantly different between the rice bran group and control at 4 weeks. Each metabolite was represented by a closed, colored node extending from a metabolic pathway node, which connects to a chemical class hexagonal node. A red node represents metabolites with significantly higher expression in rice bran group at 4 weeks compared to control group ( $p \leq 0 \cdot 05$ ). A dark blue node represents metabolites with significantly lower expression in rice bran group at 4 weeks when compared to control group. Nodes colored pink or light blue represent metabolites trending towards significance with higher expression in rice bran group at 4 weeks and lower expression at 4 weeks, respectively $(0 \cdot 05 \leq p \leq 0 \cdot 1)$. Node size is proportional to fold-difference magnitude at week 4 for rice bran group compared to control group. Bolded metabolites indicate previously reported evidence for cancer chemoprevention. 
pathways affected by rice bran were urea cycle; arginine and proline ( $\mathrm{PES}=3.5$ ), leucine, isoleucine, and valine ( $\mathrm{PES}=3.3$ ), and phenylalanine and tyrosine $(\mathrm{PES}=2.3)$. The xenobiotic metabolic pathways affected were chemicals $(\mathrm{PES}=3)$, benzoate $(\mathrm{PES}=2.8$ ), and xanthine $(\mathrm{PES}=2.2$ ). The lipid metabolic pathways affected were acyl glycine fatty acids (PES=9.2) and steroids (PES=4.4).

\section{Discussion}

This study established that increased rice bran consumption for 1 month significantly modulated the plasma and urine metabolome of CRC survivors. Multiple plasma and urine metabolites were identified as potentially derived from the rice bran composition [24]. Diet-derived and endogenous metabolites that were affected by rice bran in the diet had previously reported cancer protective actions. This study showed integration of the food and nutritional metabolome using plasma and urine samples collected from a randomized controlled dietary intervention study.

\section{Plasma metabolites with colon cancer prevention properties}

In plasma of rice bran participants, the 2 metabolites (mannose and BCG) were examined due to increased fold-differences compared to control participants. Mannose is necessary to activate mannosebinding lectin (MBL), and to initiate the MBL-complement cascade that can reduce cancer cell viability [39]. The MBL complement activation pathway was increased in the blood of participants with CRC [40], supporting that dietary sources of mannose may enhance protection against CRC. BCG also showed increased fold-changes and is structurally close to $\mathrm{N}$-acetylaspartylglutamate (NAAG), a substrate for glutamate carboxypeptidase 2 (GCP2). GCP2 is a prostate specific membrane antigen (PSMA) and highly expressed in prostate cancer [41]. BCG is an inhibitor of GCP2 [42,43] and thus might be useful in the regulation, treatment, and control of cancer [43].

There is relevance to the decreased abundance of plasma lipid metabolites in the rice bran group, such as for linoleoylcholine, oleoylcholine, palmitoylcholine, DGLA, dihomo-linoleoylcarnitine (C20:2), arachidonoylcholine, dihomo-linolenoyl-choline, docosahexaenoylcholine, and benzoate. Among these, linoleoylcholine, oleoylcholine, DGLA, arachidonoylcholine, dihomo-linolenoylcholine, and docosahexaenoylcholine also showed decreased foldchanges at 4 weeks compared to baseline. The PUFAs in this list, such as linoleoylcholine, DGLA (20:3n3 or 6), dihomo-linoleoylcarnitine (C20:2), arachidonoylcholine, dihomo-linolenoyl-choline and docosahexaenoylcholine were important to consider because a high intake of $\omega$-6-PUFAs, especially in association with a low intake of $\omega-3$ PUFAs, was shown to stimulate mammary and colon carcinogenesis, as well as increase oxidative DNA damage [44], effect cell proliferation [45], and alter free estrogen levels and hormone catabolism [46]. Mostafa et al. reported low concentrations of serum PUFAs as a manifestation of gastrointestinal problems [47]. DGLA is a unique $\omega-6$ PUFA with antiinflammatory and anti-proliferative properties [48]. DGLA competes with arachadonic acid for cyclooxygenase (COX) and lipoxygenase, inhibiting the production of eicosanoids to reduce inflammation and pain $[49,50]$. DGLA acts by inhibiting motility and invasiveness of human colon cancer cells by increasing the expression of E-cadherin, a cell-to-cell adhesion molecule that acts as a suppressor of metastasis $[51,52]$. In addition, DGLA reduces tumor-endothelium adhesion, a key factor in the establishment of distant metastases, partly by improving gap junction communication within the endothelium [51,53]. A linkage to the gut-brain axis comes from a report of higher serum DGLA concentrations decreasing risk of depression in elderly men [54]. Benzoic acid was shown to have broad spectrum bacteriostatic and fungistatic properties [55]. A recent study demonstrated that benzoic acid and its naturally occurring derivatives inhibit cancer cell growth by targeting histone deacetylase, a key enzyme in tumor regulatory gene expression [56]. These metabolites merit further investigation in people at risk that will consume rice bran for longer time periods.

\section{Urine metabolites with cancer control and prevention properties}

Urine metabolites that had increased fold-differences following rice bran consumption, and that have reported anti-cancer properties included 3-(3-hydroxyphenyl)propionate, DHEA-S, androstenediol $(3 \beta, 17 \beta)$ monosulfate, and $\mathrm{N}$-formylphenylalanine. 3-(3-hydroxyphenyl)propionate is a microbial metabolite of ingested caffeic acid, and a product of the phenolic degradation in the colon [57]. 3-(3-hydroxyphenyl)propionate has antioxidative properties and is actively transported by the monocarboxylic acid transporter in human epithelial colorectal adenocarcinoma cells (Caco-2) monolayers [57,58]. DHEA-S is the sulfated form of dehydroepiandrosterone, a natural steroid hormone produced by the adrenal glands [59]. DHEA-S has antioxidant properties and aids in inhibition of weight gain and cellular proliferation, which could contribute to the cancer protective potential $[60,61]$, and DHEA-S was shown to reduce colon cancer incidence in various animal models [61]. Androstenediol $(3 \beta, 17 \beta)$ monosulfate or in short, 5-androstenediol, can bind to estrogen receptors to suppress the proliferation signaling pathways in the mammary tumor cells [62]. $\mathrm{N}$-formylphenylalanine, a metabolite of phenylalanine metabolism showed antitumor activity in Dunning Leukemia rats [63].

$\mathrm{N}$-formylphenylalanine and 3-(4-hydroxyphenyl)lactate were identified in the rice bran food metabolome. 3-(4-hydroxyphenyl) lactate, a tyrosine metabolite, was significantly decreased in urine of CRC participants in the rice bran group at 4 weeks compared to control. 3-(4-hydroxyphenyl)lactate is also a bacterial metabolite of nonabsorbed tyrosine in intestinal lumen. High urinary concentration of this metabolite was found in individuals with tyrosine malabsorption and bacterial overgrowth $[64,65]$. Observing a decreased fold-difference of 3-(4-hydroxyphenyl)lactate herein may signify improved tyrosine absorption and balanced gut microflora that occurs with rice bran consumption and for providing protection against CRC progression in people at high risk for recurrence.

\section{Study limitations}

The important study limitations to consider in these outcomes were the small cohort size and short study duration of the diet intervention. However, the sample size $(\mathrm{N}=19)$ is comparable with other interventions using metabolomics approaches [66]. There was complexity in discerning the number of metabolic pathways and metabolites affected by a diet rich in rice bran as the participants were free-living individuals and did consume 1-2 meals/day that were different from the study-provided meals and snacks. The diversity of food compounds which yield to the presence of food metabolites that were not found in rice bran, but present in plasma and urine merit follow up attention in the overall dietary pattern. Future investigations should consider a larger sample size and longer duration of rice bran intake to observe efficacy of rice bran intake for colon cancer control mechanisms and prevention of recurrence. 


\section{Conclusion}

Daily consumption of rice bran for 1 month favorably modulates the plasma and urine metabolome and metabolites identified from rice bran had supporting evidence for metabolic mechanisms involved in colon cancer control and prevention. The benefits of integrating the host plasma and urine metabolome with rice bran food metabolite analysis allowed for direct insights into the complex interactions between food, host and gut microbial metabolism. This nutritional metabolomics approach also suggests potential utility for the identification of dietary biomarkers that link rice bran intake to long-term health benefits. The health promoting, nutritional and medicinal properties of rice bran bioactive compounds merit further investigation as a combination functional food approach to improve intestinal health, especially with regards to CRC control and prevention.

\section{Declarations}

Ethics: This study was carried out in accordance with the recommendations of local and national guidelines and all human study protocols were approved by Colorado State University and University of Colorado Health-North Institutional Review Boards. Prior to the start of the trial, written informed consent was obtained from all participants in accordance with the Declaration of Helsinki. This trial was registered at clinicaltrials.gov under NCT01929122.

Financial support: National Institutes Health - National Cancer Institute (1R21CA161472), National Institute of Food and Agriculture (2016-67001-24538) and the University of Colorado Cancer Center Division of Cancer Prevention and Control.

Conflicts of interest: No potential conflict of interest relevant to this article was reported.

Disclosure: EPR, RJB and ECB designed and conducted the research; IZ, RCO, and EPR conducted the metabolome analysis and wrote the manuscript. All authors read and approved the final manuscript.

Acknowledgements: Funding for this study was provided by National Institutes Health - National Cancer Institute (1R21CA161472), National Institute of Food and Agriculture (NIFA) (2016-6700124538), the University of Colorado Cancer Center - Division of Cancer Prevention and Control pilot award program. The authors appreciate the collaboration of University of Colorado Health-North Cancer Clinical Research group.

\section{References}

1. WCRF (2012) Colorectal cancer statistics.

2. Siegel RL, Miller KD, Jemal A (2017) Cancer statistics, 2017. CA Cancer J Clin 67: 7-30. [Crossref]

3. Slavin JL (2005) Dietary fiber and body weight. Nutrition 21: 411-418. [Crossref]

4. Howarth NC, Saltzman E, Roberts SB (2001) Dietary fiber and weight regulation. Nutr Rev 59: 129-139. [Crossref]

5. Bazzano LA, He J, Ogden LG, Loria CM, Whelton PK, et al. (2003) Dietary fiber intake and reduced risk of coronary heart disease in US men and women: The national health and nutrition examination survey 1 epidemiologic follow-up study. Arch Intern Med 163: 1897-904. [Crossref]

6. Mozaffarian D, Kumanyika SK, Lemaitre RN, Olson JL, Burke GL, et al. (2003) Cereal, fruit, and vegetable fiber intake and the risk of cardiovascular disease in elderly individuals. JAMA 289: 1659-1666. [Crossref]

7. Anderson JW, Allgood LD, Turner J, Oeltgen PR, Daggy BP (1999) Effects of psyllium on glucose and serum lipid responses in men with type 2 diabetes and hypercholesterolemia. Am J Clin Nutr 70: 466-473. [Crossref]
8. Chandalia M, Garg A, Lutjohann D, von Bergmann K, Grundy SM, et al. (2000) Beneficial effects of high dietary fiber intake in patients with type 2 diabetes mellitus. $N$ Engl J Med 342: 1392-1398. [Crossref]

9. Giacco R, Parillo M, Rivellese AA, Lasorella G, Giacco A, et al. (2000) Long-term dietary treatment with increased amounts of fiber-rich low-glycemic index natural foods improves blood glucose control and reduces the number of hypoglycemic events in type 1 diabetic patients. Diabetes Care 23: 1461-1466. [Crossref]

10. Cummings JH, Bingham SA, Heaton KW, Eastwood MA (1992) Fecal weight, colon cancer risk, and dietary intake of nonstarch polysaccharides (dietary fiber). Gastroenterology 103: 1783-1789. [Crossref]

11. Howe GR, Benito E, Castelleto R, Cornée J, Estève J, et al. (1992) Dietary intake of fiber and decreased risk of cancers of the colon and rectum: evidence from the combined analysis of 13 case-control studies. J Natl Cancer Inst 84: 1887-1896. [Crossref]

12. Kim YI (2000) AGA technical review: impact of dietary fiber on colon cancer occurrence. Gastroenterology 118: 1235-1257. [Crossref]

13. Bingham SA, Day NE, Luben R, Ferrari P, Slimani N, et al. (2003) Dietary fibre in food and protection against colorectal cancer in the European Prospective Investigation into Cancer and Nutrition (EPIC): An observational study. Lancet 361: 1496-1501. [Crossref]

14. World Cancer Research Fund, AICR (2011) Food, nutrition, physical activity, and the prevention of cancer: A global perspective. Washington DC: AICR.

15. Borresen EC, Brown DG, Harbison G, Taylor L, Fairbanks A, et al. (2016) A randomized controlled trial to increase navy bean or rice bran consumption in colorectal cancer survivors. Nutr Cancer 68: 1269-1280. [Crossref]

16. Tan XW, Bhave M, Fong AY, Matsuura E, Kobayashi K, et al. (2016) Cytoprotective and cytotoxic effects of rice bran extracts in rat $\mathrm{H} 9 \mathrm{c} 2(2-1)$ cardiomyocytes. Oxid Med Cell Longev 2016: 6943053. [Crossref]

17. Mentor-Marcel RA, Bobe G, Barrett KG, Young MR, Albert PS, et al. (2009) Inflammation-associated serum and colon markers as indicators of dietary attenuation of colon carcinogenesis in ob/ob mice. Cancer Prev Res (Phila) 2: 60-69. [Crossref]

18. Justo ML, Claro C, Zeyda M, Stulnig TM, Herrera MD, et al. (2016) Rice bran prevents high-fat diet-induced inflammation and macrophage content in adipose tissue. Eur $J$ Nutr 55: 2011-2019. [Crossref]

19. Fukuoka D, Okahara F, Hashizume K, Yanagawa K, Osaki N, et al. (2014) Triterpene alcohols and sterols from rice bran lower postprandial glucose-dependent insulinotropic polypeptide release and prevent diet-induced obesity in mice. J Appl Physiol (1985) 117: 1337-1348. [Crossref]

20. Insuan O, Chariyakornkul A, Rungrote $Y$, Wongpoomchai R (2017) Antimutagenic and Antioxidant Activities of Thai Rice Brans. J Cancer Prev 22: 89-97. [Crossref]

21. Somintara S, Leardkamolkarn V, Suttiarporn P, Mahatheeranont S (2016) Anti-tumor and immune enhancing activities of rice bran gramisterol on acute myelogenous leukemia. PLoS One 11: e146869. [Crossref]

22. Norris L, et al. (2015) Search for novel circulating cancer chemopreventive biomarkers of dietary rice bran intervention in Apc(Min) mice model of colorectal carcinogenesis, using proteomic and metabolic profiling strategies. Mol Nutr Food Res 59: 1827-1836. [Crossref]

23. Jariwalla RJ (2001) Rice-bran products: phytonutrients with potential applications in preventive and clinical medicine. Drugs Exp Clin Res 27: 17-26. [Crossref]

24. Zarei I, Brown DG, Nealon NJ, Ryan EP (2017) Rice bran metabolome contains amino acids, vitamins and cofactors, and phytochemicals with medicinal and nutritional properties. Rice (NY) 10: 24. [Crossref]

25. Brown DG, Borresen EC, Brown RJ, Ryan EP (2017) Heat-stabilised rice bran consumption by colorectal cancer survivors modulates stool metabolite profiles and metabolic networks: A randomised controlled trial. Br J Nutr 117: 1244-1256. [Crossref]

26. Sheflin AM, Borresen EC, Kirkwood JS, Boot CM, Whitney AK, et al. (2017) Dietary supplementation with rice bran or navy bean alters gut bacterial metabolism in colorectal cancer survivors. Mol Nutr Food Res 61. [Crossref]

27. Sheflin AM, Borresen EC, Wdowik MJ, Rao S, Brown RJ, et al. (2015) Pilot dietary intervention with heat-stabilized rice bran modulates stool microbiota and metabolites in healthy adults. Nutrients 7: 1282-1300. [Crossref]

28. Tantamango YM, Knutsen SF, Beeson WL, Fraser G, Sabate J (2011) Foods and food groups associated with the incidence of colorectal polyps: the Adventist Health Study. Nutr Cancer 63: 565-572. [Crossref] 
29. Henderson AJ, Ollila CA, Kumar A, Borresen EC, Raina K, et al. (2012) Chemopreventive properties of dietary rice bran: Current status and future prospects. Adv Nutr 3: 643-653. [Crossref]

30. Ajitha MJ, Mohanlal S, Suresh CH, Jayalekshmy A (2012) DPPH radical scavenging activity of tricin and its conjugates isolated from "Njavara" rice bran: A density functional theory study. J Agric Food Chem 60: 3693-3699. [Crossref]

31. Borresen EC, Jenkins-Puccetti N, Schmitz K, Brown DG, Pollack A, et al. (2017) A pilot randomized controlled clinical trial to assess tolerance and efficacy of navy bean and rice bran supplementation for lowering cholesterol in children. Glob Pediatr Health 4: 2333794X17694231. [Crossref]

32. Scalbert A, Brennan L, Manach C, Andres-Lacueva C, Dragsted LO, et al. (2014) The food metabolome: A window over dietary exposure. Am J Clin Nutr 99: 1286-1308. [Crossref]

33. O'Sullivan A, Gibney MJ, Brennan L (2011) Dietary intake patterns are reflected in metabolomic profiles: potential role in dietary assessment studies. Am J Clin Nutr 93: 314-321. [Crossref]

34. Fav G, Beckmann ME, Draper JH, Mathers JC (2009) Measurement of dietary exposure: A challenging problem which may be overcome thanks to metabolomics? Genes Nutr 4: 135-141. [Crossref]

35. Playdon MC, Sampson JN, Cross AJ, Sinha R, Guertin KA, et al. (2016) Comparing metabolite profiles of habitual diet in serum and urine. Am J Clin Nutr 104: 776-789. [Crossref]

36. Borresen EC, Gundlach KA, Wdowik M, Rao S, Brown RJ, et al. (2014) Feasibility of increased navy bean powder consumption for primary and secondary colorectal cancer prevention. Curr Nutr Food Sci 10: 112. [Crossref]

37. Brown DG, Rao S, Weir TL, O'Malia J, Bazan M, et al. (2016) Metabolomics and metabolic pathway networks from human colorectal cancers, adjacent mucosa, and stool. Cancer Metab 4: 11. [Crossref]

38. Li KJ, Borresen EC, Jenkins-Puccetti N, Luckasen G, Ryan EP (2018) Navy bean and rice bran intake alters the plasma metabolome of children at risk for cardiovascular disease. Front Nutr 4: 71. [Crossref]

39. Holmskov UL (2000) Collectins and collectin receptors in innate immunity. APMIS Suppl 100: 1-59. [Crossref]

40. Ytting H, Jensenius JC, Christensen IJ, Thiel S, Nielsen HJ (2004) Increased activity of the mannan-binding lectin complement activation pathway in patients with colorectal cancer. Scand J Gastroenterol 39: 674-679. [Crossref]

41. Collard F, Vertommen D, Constantinescu S, Buts L, Van Schaftingen E (2011) Molecular identification of beta-citrylglutamate hydrolase as glutamate carboxypeptidase 3. J Biol Chem 286: 38220-38230. [Crossref]

42. Collard F, troobant V, Lamosa P, Kapanda CN, Lambert DM, et al. (2010) Molecular identification of $\mathrm{N}$-acetylaspartylglutamate synthase and beta-citrylglutamate synthase. J Biol Chem 285: 29826-29833. [Crossref]

43. Mesters JR, Barinka C, Li W, Tsukamoto T, Majer P, et al. (2006) Structure of glutamate carboxypeptidase II, a drug target in neuronal damage and prostate cancer. EMBO J 25 1375-1384. [Crossref]

44. Bueno-de-Mesquita H, González C (1997) Main hypotheses on diet and cancer investigated in the EPIC Study. European prospective investigation into cancer and nutrition. Eur J Cancer Prev 6: 107. [Crossref]

45. Fernández-Bañares F, Esteve M, Navarro E, Cabré E, Boix J, et al. (1996) Changes of the mucosal $\mathrm{n} 3$ and $\mathrm{n} 6$ fatty acid status occur early in the colorectal adenoma-carcinoma sequence. Gut 38: 254-259. [Crossref]

46. Bartsch H, Nair J, Owen RW (1999) Dietary polyunsaturated fatty acids and cancers of the breast and colorectum: emerging evidence for their role as risk modifiers. Carcinogenesis 20: 2209-2218. [Crossref]

47. Mostafa GA, Al-Ayadhi LY (2015) Reduced levels of plasma polyunsaturated fatty acids and serum carnitine in autistic children: relation to gastrointestinal manifestations. Behav Brain Funct 11: 4. [Crossref]
48. Wang X, Lin H, Gu Y (2012) Multiple roles of dihomo- $\gamma$-linolenic acid against proliferation diseases. Lipids Health Dis 11: 25-25. [Crossref]

49. Nasrollahzadeh J, Siassi F, Doosti M, Eshraghian MR, Shokri F, et al. (2008) The influence of feeding linoleic, gamma-linolenic and docosahexaenoic acid rich oils on rat brain tumor fatty acids composition and fatty acid binding protein 7 mRNA expression. Lipids Health Dis 7: 45. [Crossref]

50. Ruan KH, Cervantes V, So S (2009) Engineering of a novel hybrid enzyme: an antiinflammatory drug target with triple catalytic activities directly converting arachidonic acid into the inflammatory prostaglandin E2. Protein Eng Des Sel 22: 733-740. [Crossref]

51. Watkins G, Martin TA, Bryce R, Mansel RE, Jiang WG (2005) Gamma-Linolenic acid regulates the expression and secretion of SPARC in human cancer cells. Prostaglandins Leukot Essent Fatty Acids 72: 273-278. [Crossref]

52. Jiang WG, Singhrao SK, Hiscox S, Hallett MB, Bryce RP, et al. (1997) Regulation of desmosomal cell adhesion in human tumour cells by polyunsaturated fatty acids. Clin Exp Metastasis 15: 593-602. [Crossref]

53. Menendez JA, Ropero S, del Barbacid MM, Montero S, Solanas M, et al. (2002) Synergistic interaction between vinorelbine and gamma-linolenic acid in breast cancer cells. Breast Cancer Res Treat 72: 203-219. [Crossref]

54. Yary T, et al. (2017) Serum dihomo-gamma-linolenic acid level is inversely associated with the risk of depression. A 21 -year follow-up study in general population men. $J$ Affect Disord 213: 151-155. [Crossref]

55. Navratil M, Tykvart J, Schimer J, Pachl P, Navrátil V, et al. (2016) Comparison of human glutamate carboxypeptidases II and III reveals their divergent substrate specificities. FEBS J 283: 2528-2545. [Crossref]

56. Anantharaju PG, Reddy BD, Padukudru MA, Kumari Chitturi CM, Vimalambike MG, et al. (2017) Naturally occurring benzoic acid derivatives retard cancer cell growth by inhibiting histone deacetylases (HDAC). Cancer Biol Ther 18: 492-504. [Crossref]

57. Konishi Y, Kobayashi S (2004) Microbial metabolites of ingested caffeic acid are absorbed by the monocarboxylic acid transporter (MCT) in intestinal Caco-2 cell monolayers. J Agric Food Chem 52: 6418-6424. [Crossref]

58. Rios LY, Gonthier MP, Rémésy C, Mila I, Lapierre C, et al. (2003) Chocolate intake increases urinary excretion of polyphenol-derived phenolic acids in healthy human subjects. Am J Clin Nutr 77: 912-918. [Crossref]

59. Prough RA, Clark BJ, Klinge CM (2016) Novel mechanisms for DHEA action. J Mo Endocrinol 56: R139-155. [Crossref]

60. Schwartz AG, Pashko LL (1995) Cancer prevention with dehydroepiandrosterone and non-androgenic structural analogs. J Cell Biochem Suppl 22: 210-217.

61. Fettman MJ, Butler RN, McMichael AJ, Roberts-Thomson IC (1991) Metabolic phenotypes and colorectal neoplasia. J Gastroenterol Hepatol 6: 81-89. [Crossref]

62. Bird CE, Masters V, Sterns EE, Clark AF (1982) Effects of aminoglutethimide on delta 5 -androstenediol metabolism in postmenopausal women with breast cancer. Cancer Res 42: 4797-4800. [Crossref]

63. Skinner WA, Hyde KA, Gram HF, Baker BR (1960) Potential anticancer agents ${ }^{1}$ XXXVIII. Alkylating agents related to phenylalanine mustard. II. J Org Chem 25 : $1756-1759$

64. Spaapen LJ, Ketting D, Wadman SK, Bruinvis L, Duran M (1987) Urinary D-4 hydroxyphenyllactate, D-phenyllactate and D-2-hydroxyisocaproate, abnormalities of bacterial origin. J Inherit Metab Dis 10: 383-390. [Crossref]

65. Beloborodova N, Bairamov I, Olenin A, Shubina V, Teplova V, et al. (2012) Effect of phenolic acids of microbial origin on production of reactive oxygen species in mitochondria and neutrophils. J Biomed Sci 19: 89. [Crossref]

66. Walsh MC, Brennan L, Malthouse JP, Roche HM, Gibney MJ (2006) Effect of acute dietary standardization on the urinary, plasma, and salivary metabolomic profiles of healthy humans. Am J Clin Nutr 84: 531-539. [Crossref]

Copyright: (C2019 Zarei I. This is an open-access article distributed under the terms of the Creative Commons Attribution License, which permits unrestricted use, distribution, and reproduction in any medium, provided the original author and source are credited. 